Portland State University

PDXScholar

\title{
Spontaneous Parametric Down-Conversion and Quantum Entanglement
}

Jesse Catalano

Portland State University

Follow this and additional works at: https://pdxscholar.library.pdx.edu/honorstheses Let us know how access to this document benefits you.

\section{Recommended Citation}

Catalano, Jesse, "Spontaneous Parametric Down-Conversion and Quantum Entanglement" (2014).

University Honors Theses. Paper 474.

https://doi.org/10.15760/honors.474

This Thesis is brought to you for free and open access. It has been accepted for inclusion in University Honors Theses by an authorized administrator of PDXScholar. Please contact us if we can make this document more accessible: pdxscholar@pdx.edu. 
Spontaneous Parametric Down-Conversion and Quantum Entanglement

\author{
by \\ Jesse Catalano
}

An undergraduate honors thesis submitted in partial fulfillment of the

\author{
requirements for the degree of \\ Bachelor of Science \\ in \\ University Honors \\ and
}

Physics

Thesis Adviser

Andres La Rosa

Portland State University 


\section{Table of Contents}

Introduction.

1.1 Classical Theory of Spontaneous Parametric Down-Conversion (SPDC) Introduction.

1.2 The Wave Equation..........................................................5

1.3 Ordinary and Extraordinary Polarization Directions............................8

1.4 Non-linear Polarization and the $[d]$ Matrix....................................9

1.5 Coupled Equations for Type-I and Type-II SPDC.............................11

2.1 Polarization Entanglement for Type-I and Type-II SPDC.....................13

2.2 Bell Inequality and Local-realism.........................................15

3.1 Quantum Teleportation using Entangled Photons................................19

3.2 Entanglement Transfer from Photons to Matter...............................21

Conclusion..............................................................................23

Acknowledgments.......................................................................24

Appendix - Vector Orientation Relative to the Principle Axis.......................24

References..............................................................................25 


\section{Abstract}

Quantum entanglement has been a topic of much research in modern physics, and an application in quantum computing is envisioned. This thesis address the use of polarization entangled photon pairs produced by spontaneous parametric down-conversion (SPDC) in entanglement transfer process and quantum computing. The simplicity of SPDC entanglement relative to other quantum phenomena is first emphasized by developing a complete classical theory of the photon-crystal interactions characteristic of SPDC. The methods of achieving polarization superposition via the Type-I and Type-II SPDC are described. A combination of superposition and polarization correlation between downconverted photons is shown to violate Bell's inequality, thus revealing the entangled nature of the photon pairs. Finally, the possibility of controlling entangled photons through the manipulation of surface plasmons is highlighted as a way to transfer entanglement from photons to material particles.

\section{Introduction}

Spontaneous parametric down-conversion (SPDC) is a $2^{\text {nd }}$ order optical effect where the dipole polarization depends quadratically on the electric field and photons incident on a non-linear crystal can be "converted" into two photons. It is an important process in quantum optics, used especially to produce entangled photon pairs ${ }^{1}$, and also constitutes an excellent method to produce a source of single photons ${ }^{2}$. An incident photon in SPDC is often referred to as the "pump" while the outgoing photons are called the "signal" and "idler". SPDC is said to be "spontaneous" because there is no input signal or idler field to stimulate the process; they are generated spontaneously inside the crystal. The process is "parametric" because it depends on the electric fields (and not just their intensities), implying that there exists a phase relationship between input and output fields. "Down-conversion" refers to that fact that the signal and idler fields always have a lower frequency than the pump. ${ }^{3}$ Splitting of the pump photons into pairs of photons occurs in accordance with conservation laws, namely, having combined energies and momenta equal to the energy and momentum of the original photon. These conservation laws are given by

$$
\begin{aligned}
& \omega_{3}=\omega_{1}+\omega_{2} \\
& \boldsymbol{k}_{3}=\boldsymbol{k}_{1}+\boldsymbol{k}_{2},
\end{aligned}
$$

where $\omega_{3}, \omega_{1}, \omega_{2}, \boldsymbol{k}_{3}, \boldsymbol{k}_{1}$, and $\boldsymbol{k}_{2}$, are the frequencies and wave vectors of the pump (subscript 3 ), signal (subscript 1), and idler (subscript 2).

SPDC, as analyzed in this paper, is a phenomenon that requires photon-crystal interactions among the three photons within a uniaxial crystal, where the pump photon is partially or fully polarized in an extraordinary direction relative to the crystal's optical axis. SPDC can be implemented in two different varieties, characterized by the polarizations of the signal and idler. If both outgoing photons are ordinary in their polarization it is deemed Type-I down-conversion. If one of the photons is extraordinarily polarized while the other is ordinary, it is called Type-II. The possible paths of the outgoing photons (schematically shown in Fig. 1) are determined by conservation laws and constraints on polarization. In either case, the outgoing photons are correlated in their polarizations.

The direction of propagation for the signal and idler waves depend upon several factors, such as their respective frequencies, the ordinary and extraordinary refractive indexes of the non-linear crystal, $\mathrm{n}_{\mathrm{o}}$ and $\mathrm{n}_{\mathrm{e}}$, the angle between the pump and the crystal axis, and the conservation laws (1a) and (1b). Additionally, the strength of the down-converted fields is determined by phase matching conditions ${ }^{4}$, which are dependent upon crystal thickness and propagation speeds. Because propagation speeds depend upon indices of refraction, Type-I and Type-II SPDC have different phase matching conditions. 
Type-I Down-Conversion

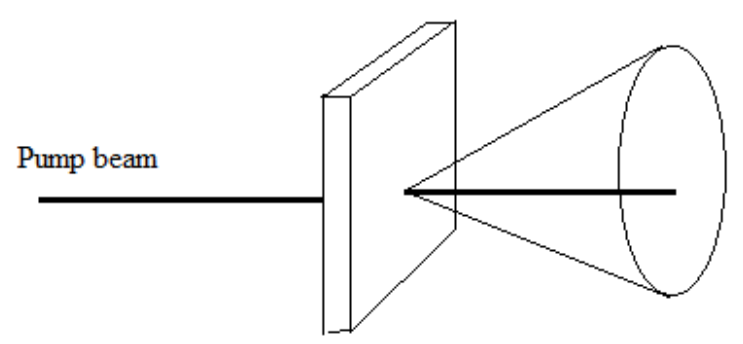

Type-II Down-Conversion

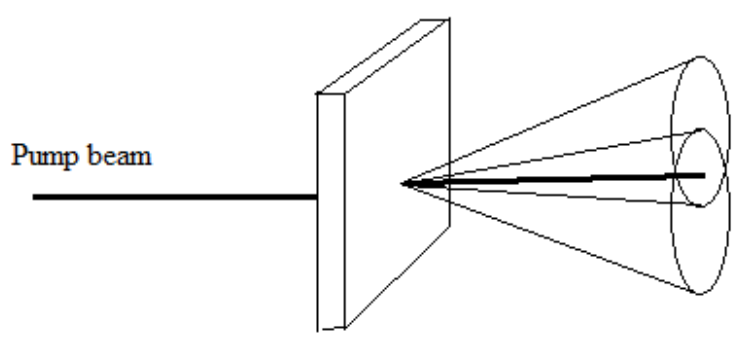

Fig 1: The two types of Parametric Down-Conversion from a $2^{\text {nd }}$ order crystal. LEFT: Type-I down-conversion, where the pump beam is converted into two beams with paths constrained to a cone centered on the pump. The output photons have ordinary polarization relative to the crystal axis. RIGHT: Type-II down-conversion, where the pump beam is converted into two beams which each appear anywhere on their respective cones, on opposite sides of the pump beam. One of the outgoing photons will be ordinarily polarized, while the other will be extraordinary.

Both types can be attained by tuning the orientation of the non-linear crystal relative to the incident beam direction. For example, in one experiment, Type-I SPDC was attained using a $413 \mathrm{~nm}$ pump beam propagating at $28.3^{\circ}$ from the crystal axis of a $1 \mathrm{~mm}$ thick BBO crystal $(\beta$-barium borate, $\beta$ $\left.\mathrm{BaB}_{2} \mathrm{O}_{4}\right)^{5}$. In another experiment, Type-II SPDC was attained using a $341 \mathrm{~nm}$ pump at $49.6^{\circ}$ from the crystal axis of a $3 \mathrm{~mm}$ thick BBO crystal $^{6}$.

The correlation between down-converted photon polarization states makes possible the production of polarization-entangled photons. Entanglement is the quantum phenomenon where the state a system of two or more particles is not determined by the states of the individual particles alone, and only occurs when the particles of the system are in a quantum superposition of states and share a correlation. If two photons are correlated in their polarizations, measuring the polarization state of one determines the state of the other, without having to measure it. SPDC is adequate to produce photons which are both correlated and in superposition. For Type-II, the outgoing photons are in superposition where the cones intersect, because the polarization at that point is uncertain. For Type-I, two crystals must be aligned in such a way that their cones overlap. This will be further explained in chapter 2 of this paper.

In the first chapter of this paper, a theory of SPDC will be developed from first principles (Maxwell's equations). In chapter 2, the methods for attaining entanglement of photon polarizations for both Type-I and Type-II SPDC will be given. It will also be shown that correlation between downconverted photon polarizations puts into doubt local-realism, a viewpoint that considers reality as deterministic, independent of the observer, and that no information travels faster than light.

Consequences of the violation of local-realism will be explored. Chapter 3 presents methods of manipulating polarization entangled photons, and proposes a use for SPDC in entanglement transfer experiments.

\subsection{Classical Theory of SPDC - Introduction}

We present here a theory of SPDC based on the Maxwell equations and the concept of nonlinear polarization. This treatment leads to three coupled equations for both Type-I and Type-II downconversion. The derivation of these equations requires many steps and several approximations. It is the objective of this chapter to present a complete derivation of these coupled equations in a thorough and logical manner. Throughout this chapter, it will be assumed that the non-linear crystal through which 
SPDC occurs is $\mathrm{KDP}\left(\mathrm{KH}_{2} \mathrm{PO}_{4}\right)$. The results of this chapter can be extended to other uniaxial crystals.

\subsection{The Wave Equation}

Spontaneous parametric down-conversion (SPDC) is a $2^{\text {nd }}$ order optical effect which requires non-linear uniaxial crystals. The behavior of light in a non-linear crystal can be determined from first principles. We can begin with Maxwell's equations in matter. If we assume no electric currents or free charges are present in the medium, then the electric field $\mathbf{E}$, magnetic field $\mathbf{B}$, and electric displacement field $\mathbf{D}$, are related by

$$
\begin{aligned}
& \nabla x \boldsymbol{E}=-\frac{\partial}{\partial t} \boldsymbol{B} \\
& \nabla x \boldsymbol{B}=\mu \frac{\partial}{\partial t} \boldsymbol{D},
\end{aligned}
$$

where $\mu$ is the permeability constant of the material. Given equation (3), the curl of equation (2) is

$$
\nabla x \nabla x \boldsymbol{E}=-\mu \frac{\partial^{2}}{\partial t^{2}} \boldsymbol{D}
$$

The curl operators on the left side of equation (4) can be replaced using the following identity,

$$
\nabla x \nabla x E=\nabla(\nabla \cdot E)-\nabla^{2} E
$$

In a linear material, the polarization $\mathbf{P}$ is related to the electric field by $\mathbf{P}_{\text {linear }}=\varepsilon_{0} \chi_{\text {linear }} \mathbf{E}$, where $\varepsilon_{0}$ is the permittivity of a vacuum. Therefore, the electric displacement vector $\mathbf{D} \equiv \varepsilon_{0} \mathbf{E}+\mathbf{P}$ in a linear material can be written as $\mathbf{D} \equiv \varepsilon_{0} \mathbf{E}+\mathbf{P}=\varepsilon_{0}\left(1+\chi_{\text {linear }}\right) \mathbf{E} \equiv \varepsilon \mathbf{E}$. In the non-linear case, $\mathbf{P}=\mathbf{P}_{\text {linear }}+\mathbf{P}_{\mathrm{NL}}=\varepsilon_{0} \chi_{\text {linear }} \mathbf{E}$ $+\mathbf{P}_{\mathrm{NL}}$. Therefore, the electric displacement in a non-linear material is

$$
\boldsymbol{D} \equiv \varepsilon_{0} \boldsymbol{E}+\boldsymbol{P}=\varepsilon \boldsymbol{E}+\boldsymbol{P}_{N L}
$$

Using equations (5) and (6), equation (4) can be written as

$$
\nabla^{2} \boldsymbol{E}-\nabla(\nabla \cdot \boldsymbol{E})-\mu \varepsilon \frac{\partial^{2}}{\partial t^{2}} \boldsymbol{E}=\mu \frac{\partial^{2}}{\partial t^{2}} \boldsymbol{P}_{N L}
$$

Equation (7) is a wave equation which explicitly shows how the polarization of a non-linear crystal is related to the electric field within it. This equation holds for each vector component of any basis, hence, projecting $\mathbf{E}$ and $\mathbf{P}_{\mathrm{NL}}$ on any particular axis results in a scalar relationship, which can be replace in equation (7).

SPDC is a three-photon phenomenon, so $\mathbf{E}$ is the summation of three fields (the pump, signal, and idler). Let $\mathbf{E}$ be written as,

$$
\boldsymbol{E}=\boldsymbol{E}\left(s, t, \omega_{1}\right)+\boldsymbol{E}\left(s, t, \omega_{2}\right)+\boldsymbol{E}\left(s, t, \omega_{3}\right),
$$

with

$$
\boldsymbol{E}\left(s, t, \omega_{1}\right)=\frac{1}{2} \boldsymbol{E}_{1}(s) e^{i\left(\boldsymbol{k}_{1} \cdot \boldsymbol{r}-\omega_{1} t\right)}+c . c .
$$




$$
\begin{aligned}
& \boldsymbol{E}\left(s, t, \omega_{2}\right)=\frac{1}{2} \boldsymbol{E}_{2}(s) e^{i\left(\boldsymbol{k}_{2} \cdot \boldsymbol{r}-\omega_{2} t\right)}+c . c . \\
& \boldsymbol{E}\left(s, t, \omega_{3}\right)=\frac{1}{2} \boldsymbol{E}_{3}(s) e^{i\left(\boldsymbol{k}_{3} \cdot \boldsymbol{r}-\omega_{3} t\right)}+c . c .
\end{aligned}
$$

where $\mathrm{s}$ is the distance propagated through the crystal and c.c. is the complex conjugate of the preceding term.

The amplitudes from equations (8a-c) are complex values containing phase information. Note that we allow for the amplitudes to change as the waves propagate. This is because in SPDC, some of the energy of the pump beam represented here as the third wave with frequency $\omega_{3}$, gets "converted" into the other two waves. Therefore the pump beam decreases in intensity along the crystal length.We are assuming that none of the three amplitudes have components in the direction of propagation $\hat{\boldsymbol{s}}$. In reality, the signal and idler are usually measured along a very small angle relative to the pump direction of propagation.

Using equation (7), we want to determine the relationship between electric fields and polarizations that are functions oscillating at frequencies $\omega_{1}, \omega_{2}$, and $\omega_{3}$. Equation (7) is a linear equation, so each term of $\mathbf{E}$ can be differentiated separately, the results being added together afterwards to attain the left side of equation (7). Let us focus on the pump. If the pump is defined as in (8c), then

$$
\begin{aligned}
& \nabla^{2} \boldsymbol{E}\left(s, t, \omega_{3}\right)=\left[-k_{3}{ }^{2} \boldsymbol{E}_{3}+2 i k_{3, s} \frac{d}{d s} \boldsymbol{E}_{3}+\frac{\partial^{2}}{\partial s^{2}} \boldsymbol{E}_{3}\right]\left(\frac{1}{2} e^{i\left(\boldsymbol{k}_{3} \cdot \boldsymbol{r}-\omega_{3} t\right)}\right)+c . c . \\
& -\nabla\left(\nabla \cdot \boldsymbol{E}\left(s, t, \omega_{3}\right)\right)=\left[\left(\boldsymbol{k}_{3} \cdot \boldsymbol{E}_{3}\right) \boldsymbol{k}_{3}-i\left(\boldsymbol{k}_{3} \cdot \frac{d}{d s} \boldsymbol{E}_{3}\right) \hat{\boldsymbol{s}}\right]\left(\frac{1}{2} e^{i\left(\boldsymbol{k}_{3} \cdot \boldsymbol{r}-\omega_{3} t\right)}\right)+c . c . \\
& -\mu \varepsilon \frac{\partial^{2}}{\partial t^{2}} \boldsymbol{E}\left(s, t, \omega_{3}\right)=\mu \varepsilon \omega_{3}{ }^{2} \boldsymbol{E}_{3}\left(\frac{1}{2} e^{i\left(\boldsymbol{k}_{3} \cdot \boldsymbol{r}-\omega_{3} t\right)}\right)+c . c .
\end{aligned}
$$

where $\mathrm{k}_{3, \mathrm{~S}}$ is the component of $\mathbf{k}_{3}$ in the direction of propagation $\hat{\boldsymbol{s}}, \mathrm{k}_{3}$ is the absolute value of $\mathbf{k}_{3}$, and c.c. is the complex conjugate of the preceding terms. The sum of these terms is

$$
\nabla^{2} \boldsymbol{E}\left(s, t, \omega_{3}\right)-\nabla\left(\nabla \cdot \boldsymbol{E}\left(s, t, \omega_{3}\right)\right)-\mu \varepsilon \frac{\partial^{2}}{\partial t^{2}} \boldsymbol{E}\left(s, t, \omega_{3}\right)=\boldsymbol{A}_{3} e^{i\left(\boldsymbol{k}_{3} \cdot \boldsymbol{r}-\omega_{3} t\right)}+c . c .
$$

where

$$
\boldsymbol{A}_{3}=\frac{1}{2}\left(-k_{3}{ }^{2} \boldsymbol{E}_{3}+\frac{\partial^{2}}{\partial s^{2}} \boldsymbol{E}_{3}+2 i k_{3, s} \frac{d}{d s} \boldsymbol{E}_{3}-i\left(\boldsymbol{k}_{3} \cdot \frac{d}{d s} \boldsymbol{E}_{3}\right) \hat{\boldsymbol{s}}+\left(\boldsymbol{k}_{3} \cdot \boldsymbol{E}_{3}\right) \boldsymbol{k}_{3}+\mu \varepsilon \omega_{3}{ }^{2} \boldsymbol{E}_{3}\right),
$$

where the complex conjugate is omitted for convenience. Two more equations similar to (9a) exist for both $\mathbf{E}\left(s, t, \omega_{1}\right)$ and $\mathbf{E}\left(s, t, \omega_{2}\right)$ respectively.

$$
\begin{aligned}
& \nabla^{2} \boldsymbol{E}\left(s, t, \omega_{1}\right)-\nabla\left(\nabla \cdot \boldsymbol{E}\left(s, t, \omega_{1}\right)\right)-\mu \varepsilon \frac{\partial^{2}}{\partial t^{2}} \boldsymbol{E}\left(s, t, \omega_{1}\right)=\boldsymbol{A}_{1} e^{i\left(\boldsymbol{k}_{1} \cdot \boldsymbol{r}-\omega_{1} t\right)}+c . c . \\
& \nabla^{2} \boldsymbol{E}\left(s, t, \omega_{2}\right)-\nabla\left(\nabla \cdot \boldsymbol{E}\left(s, t, \omega_{2}\right)\right)-\mu \varepsilon \frac{\partial^{2}}{\partial t^{2}} \boldsymbol{E}\left(s, t, \omega_{2}\right)=\boldsymbol{A}_{2} e^{i\left(\boldsymbol{k}_{2} \cdot \boldsymbol{r}-\omega_{2} t\right)}+c . c .
\end{aligned}
$$

where

$$
\boldsymbol{A}_{1}=\frac{1}{2}\left(-k_{1}{ }^{2} \boldsymbol{E}_{1}+\frac{\partial^{2}}{\partial s^{2}} \boldsymbol{E}_{1}+2 i k_{1, s} \frac{d}{d s} \boldsymbol{E}_{3}-i\left(\boldsymbol{k}_{1} \cdot \frac{d}{d s} \boldsymbol{E}_{1}\right) \hat{\boldsymbol{s}}+\left(\boldsymbol{k}_{1} \cdot \boldsymbol{E}_{1}\right) \boldsymbol{k}_{1}+\mu \varepsilon \omega_{1}{ }^{2} \boldsymbol{E}_{1}\right)
$$




$$
\boldsymbol{A}_{2}=\frac{1}{2}\left(-k_{2}^{2} \boldsymbol{E}_{2}+\frac{\partial^{2}}{\partial s^{2}} \boldsymbol{E}_{2}+2 i k_{2, s} \frac{d}{d s} \boldsymbol{E}_{2}-i\left(\boldsymbol{k}_{2} \cdot \frac{d}{d s} \boldsymbol{E}_{2}\right) \hat{\boldsymbol{s}}+\left(\boldsymbol{k}_{2} \cdot \boldsymbol{E}_{2}\right) \boldsymbol{k}_{2}+\mu \varepsilon \omega_{2}^{2} \boldsymbol{E}_{2}\right)
$$

The left side of equation (7) is the sum of equations (8a-c).

$$
\nabla^{2} \boldsymbol{E}-\nabla(\nabla \cdot \boldsymbol{E})-\mu \varepsilon \frac{\partial^{2}}{\partial t^{2}} \boldsymbol{E}=\boldsymbol{A}_{1} e^{i\left(\boldsymbol{k}_{1} \cdot \boldsymbol{r}-\omega_{1} t\right)}+\boldsymbol{A}_{2} e^{i\left(\boldsymbol{k}_{2} \cdot \boldsymbol{r}-\omega_{2} t\right)}+\boldsymbol{A}_{3} e^{i\left(\boldsymbol{k}_{3} \cdot \boldsymbol{r}-\omega_{3} t\right)}
$$

Now that the form of the left side of equation (7) is known, we turn our focus to polarization in order to determine the form the right side. The $\mathrm{i}^{\text {th }}$ term of the total $2^{\text {nd }}$ order non-linear polarization is given by

$$
\left(P_{N L}\right)_{i}=\varepsilon_{0} \chi_{i j k} E_{j} E_{k}
$$

where $E_{j}$ is the $j^{\text {th }}$ component of $\mathbf{E}$, and $\chi_{i j k}$ is a component of a $3 \times 3 \times 3$ tensor, the value of which depends on the crystal and the reference frame. This formula assumes repeated summation over the indices. If $\mathbf{E}$ is the sum of the pump, signal, and idler as defined in (8a-c), then equation (11) takes the form

$$
\left(P_{N L}\right)_{i}=\varepsilon_{0} \chi_{i j k} \sum_{n}^{1,2,3}\left(\frac{1}{2} E_{n, j} e^{i\left(\boldsymbol{k}_{n} \cdot \boldsymbol{r}-\omega_{n} t\right)}+\text { c.c. }\right) \sum_{m}^{1,2,3}\left(\frac{1}{2} E_{m, k} e^{i\left(\boldsymbol{k}_{m} \cdot \boldsymbol{r}-\omega_{m} t\right)}+\text { c.c. }\right) .
$$

When equation (12) is expanded, it can be written as the sum of several polarization terms, each a function of a different frequency;

$$
\begin{aligned}
& \left(P_{N L}\right)_{i}=P_{i}\left(2 \omega_{1}\right)+P_{i}\left(2 \omega_{2}\right)+P_{i}\left(2 \omega_{3}\right)+P_{i}\left(\omega_{1}+\omega_{2}\right)+P_{i}\left(\omega_{1}+\omega_{3}\right)+P_{i}\left(\omega_{2}+\omega_{3}\right)+ \\
& P_{i}\left(\omega_{3}-\omega_{1}\right)+P_{i}\left(\omega_{3}-\omega_{2}\right)+P_{i}\left(\omega_{2}-\omega_{1}\right)+P_{i}(0) .
\end{aligned}
$$

At first glance it appears as though none of the terms in (13) are functions of $\omega_{1}, \omega_{2}$, or $\omega_{3}$. If we employ the condition of conservation of energy, from equation (1a), we see that $\omega_{3}=\omega_{1}+\omega_{2}$, $\omega_{1}=\omega_{3}-\omega_{2}$, and $\omega_{2}=\omega_{3}-\omega_{1}$. Therefore,

$$
\begin{aligned}
& P_{i}\left(\omega_{3}-\omega_{2}\right)=P_{i}\left(\omega_{1}\right) \\
& P_{i}\left(\omega_{3}-\omega_{1}\right)=P_{i}\left(\omega_{2}\right) \\
& P_{i}\left(\omega_{1}+\omega_{2}\right)=P_{i}\left(\omega_{3}\right),
\end{aligned}
$$

so, using equation (13) and (1),

$$
\begin{aligned}
& P_{i}\left(\omega_{1}\right)=B_{1, i} e^{i\left(\boldsymbol{k}_{1} \cdot \boldsymbol{r}-\omega_{1} t\right)}+c . c . \\
& P_{i}\left(\omega_{2}\right)=B_{2, i} e^{i\left(\boldsymbol{k}_{2} \cdot \boldsymbol{r}-\omega_{2} t\right)}+c . c . \\
& P_{i}\left(\omega_{3}\right)=B_{3, i} e^{i\left(\boldsymbol{k}_{3} \cdot \boldsymbol{r}-\omega_{3} t\right)}+c . c .
\end{aligned}
$$

where,

$$
\begin{aligned}
& B_{1, i}=\frac{1}{4} \varepsilon_{0} \chi_{i j k}\left(E_{3, j} E_{2, k}{ }^{*}+E_{3, k} E_{2, j}{ }^{*}\right) \\
& B_{2, i}=\frac{1}{4} \varepsilon_{0} \chi_{i j k}\left(E_{3, j} E_{1, k}{ }^{*}+E_{3, k} E_{1, j}{ }^{*}\right) \\
& B_{3, i}=\frac{1}{4} \varepsilon_{0} \chi_{i j k}\left(E_{1, j} E_{2, k}+E_{1, k} E_{2, j}\right),
\end{aligned}
$$

assuming repeated summation over the indices. As said before, we are interested in the relationship 
between electric fields and polarizations oscillating at frequencies $\omega_{1}, \omega_{2}$, and $\omega_{3}$. Therefore, the sum of equations (14a-c) should be replaced into the right side of equation (7).

$$
\begin{aligned}
& \mu \frac{\partial^{2}}{\partial t^{2}} \boldsymbol{P}\left(\omega_{1}\right)=-\mu \omega_{1}^{2} \boldsymbol{B}_{1} e^{i\left(\boldsymbol{k}_{1} \cdot \boldsymbol{r}-\omega_{1} t\right)}+c . c . \\
& \mu \frac{\partial^{2}}{\partial t^{2}} \boldsymbol{P}\left(\omega_{2}\right)=-\mu \omega_{2}^{2} \boldsymbol{B}_{2} e^{i\left(\boldsymbol{k}_{2} \cdot \boldsymbol{r}-\omega_{2} t\right)}+\text { c. c. } \\
& \mu \frac{\partial^{2}}{\partial t^{2}} \boldsymbol{P}\left(\omega_{3}\right)=-\mu \omega_{3}^{2} \boldsymbol{B}_{3} e^{i\left(\boldsymbol{k}_{3} \cdot \boldsymbol{r}-\omega_{3} t\right)}+\text { c. c. }
\end{aligned}
$$

Replacing equations (9) and (15a-c) into equation (7) results in

$$
\begin{gathered}
\boldsymbol{A}_{1} e^{i\left(\boldsymbol{k}_{1} \cdot \boldsymbol{r}-\omega_{1} t\right)}+\boldsymbol{A}_{2} e^{i\left(\boldsymbol{k}_{2} \cdot \boldsymbol{r}-\omega_{2} t\right)}+\boldsymbol{A}_{3} e^{i\left(\boldsymbol{k}_{3} \cdot \boldsymbol{r}-\omega_{3} t\right)}+\text { c.c. }=-\mu \omega_{1}{ }^{2} \boldsymbol{B}_{1} e^{i\left(\boldsymbol{k}_{1} \cdot \boldsymbol{r}-\omega_{1} t\right)}- \\
\mu \omega_{2}{ }^{2} \boldsymbol{B}_{2} e^{i\left(\boldsymbol{k}_{2} \cdot \boldsymbol{r}-\omega_{2} t\right)}-\mu \omega_{3}{ }^{2} \boldsymbol{B}_{3} e^{i\left(\boldsymbol{k}_{3} \cdot \boldsymbol{r}-\omega_{3} t\right)}+\text { c.c. }
\end{gathered}
$$

Note that because equation (16) must be true for any location $\mathbf{r}$ within the crystal at any time $t$, it must be that case that

$$
\boldsymbol{A}_{n}=-\mu \omega_{n}^{2} \boldsymbol{B}_{n} \quad \text { for } n=1,2,3 .
$$

The equations which describe the coupled relationship between pump, signal, and idler are derived from this relationship, which will be the main result of this section. Before that is done, we must be able to express equation (17) in vector format. This cannot be done in their current form because $\chi_{\mathrm{ijk}}$ are components of a $3 \times 3 \times 3$ tensor, which cannot easily be written in matrix notation. We must make use of coordinate transformations and symmetries in our equations to simplify equation (17).

\subsection{Ordinary and Extraordinary Polarization Directions}

We now define the ordinary, extraordinary, and propagation directions in terms of polar coordinates. This coordinate system will eventually allow for $\boldsymbol{B}_{n}$ to be written in its entirety in one line. Let the optical axis of the birefringent crystal be in the $\hat{\mathbf{z}}$ direction, and let the direction of the propagation of energy for the pump beam $\hat{\boldsymbol{s}}$, be oriented such that the wave is propagating at an angle $\theta$ from the $\mathrm{z}$ axis. Also, let $\hat{\boldsymbol{s}}$ be an azimuth angle $\varphi$ from the xz-plane (see Fig 2 below). The direction of propagation in terms of the principle axis is therefore

$$
\hat{\boldsymbol{s}}=\widehat{\boldsymbol{x}} \cos \varphi \sin \theta+\widehat{\boldsymbol{y}} \sin \varphi \sin \theta+\hat{\mathbf{z}} \cos \theta
$$

The ordinary direction must be perpendicular to the optical axis as well as to the direction of propagation $\hat{\boldsymbol{s}}$. This direction can be found with a cross product. As shown in Fig $2,|\hat{\boldsymbol{s}} \times \hat{\boldsymbol{z}}|=\sin \theta$, so the ordinary direction $\widehat{\boldsymbol{o}}$ must be

$$
\widehat{\boldsymbol{o}}=\frac{\hat{\boldsymbol{s}} \boldsymbol{x} \hat{\mathbf{z}}}{\sin \theta}=\widehat{\boldsymbol{x}} \sin \varphi-\widehat{\boldsymbol{y}} \cos \varphi
$$

If we define the extraordinary direction $\hat{\boldsymbol{e}}$ as being perpendicular to $\hat{\boldsymbol{s}}$ and the ordinary direction $\widehat{\boldsymbol{o}}$, then the cross product between $\widehat{\boldsymbol{o}}$ and $\widehat{\boldsymbol{s}}$ shows us that

$$
\hat{\boldsymbol{e}}=\widehat{\boldsymbol{x}} \cos \theta \cos \varphi+\widehat{\boldsymbol{y}} \cos \theta \sin \varphi-\hat{\boldsymbol{z}} \sin \theta
$$




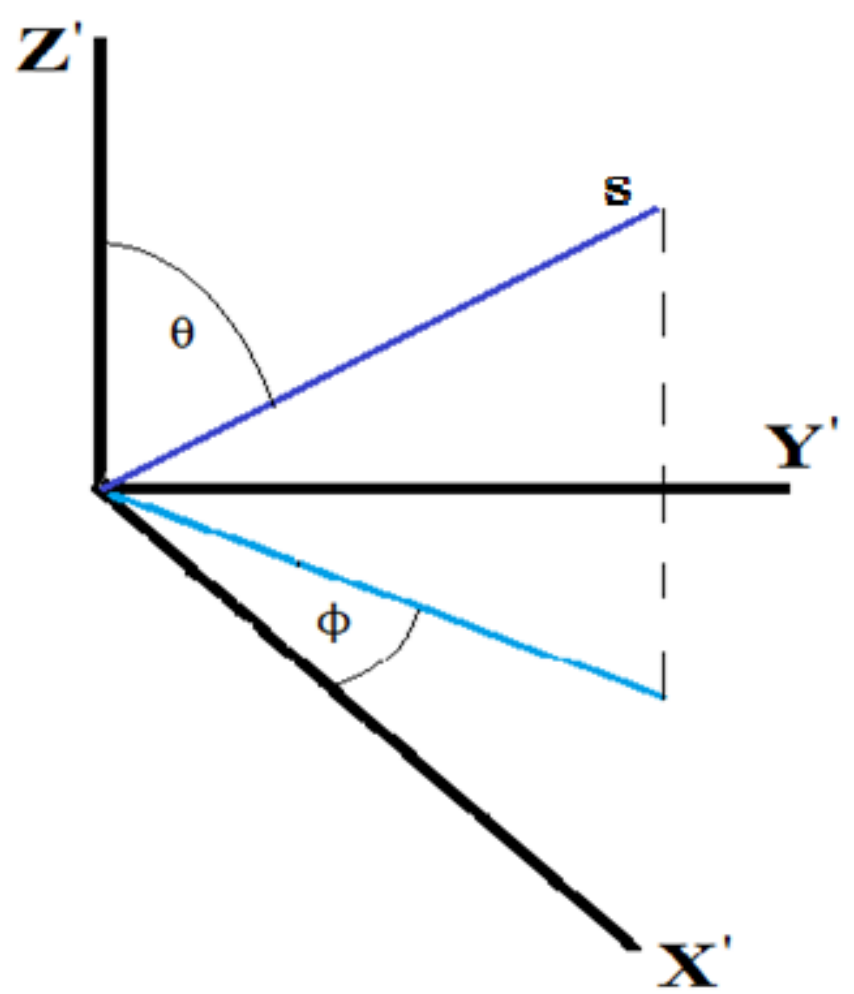

Fig 2: Diagram of wave propagating in direction $\mathbf{s}$ at angle $\theta$ from optical axis and $\varphi$ from $\mathrm{x}$-axis

Now we look at the projection of some vector $\mathbf{V}=\left(\mathrm{V}_{\mathrm{x}}, \mathrm{V}_{\mathrm{y}}, \mathrm{V}_{\mathrm{z}}\right)$ along the extraordinary direction. This projection will be used to compare the components of equation (17) in the extraordinary direction. The projection of $\mathbf{V}$ along $\hat{\boldsymbol{e}}$ is

$$
V_{\|}=V_{x} \cos \theta \cos \varphi+V_{y} \cos \theta \sin \varphi-V_{z} \sin \theta
$$

We now employ equations (18), (19) and (20) to simplify equation (17) in the case of Type-I and TypeII down-conversion in KDP crystal.

\subsection{Non-linear Polarization and the [d] Matrix}

In simplifying equation (17), we shall again focus on the pump beam, and then extend the results to the signal and idler. The $\mathrm{i}^{\text {th }}$ component of $\mathbf{B}_{3}$ is given by

$$
B_{3, i}=\frac{1}{4} \varepsilon_{0} \chi_{i j k}\left(E_{1, j} E_{2, k}+E_{1, k} E_{2, j}\right)
$$

Notice that there is no difference in the sum $E_{1, j} E_{2, k}+E_{1, k} E_{2, j}$ if the $j$ and $k$ indices are swapped $\left(E_{1, k} E_{2, j}+E_{1, j} E_{2, k}\right)$. Because it does not change the summation of these amplitudes if $j$ and $k$ are interchanged, we can define a new optical matrix $[\mathrm{d}]$ with coefficients defined in terms of $\chi_{\mathrm{ijk}}$. Let

$$
d_{i M}=\frac{1}{2}\left(\chi_{i j k}+\chi_{i j k}\right)
$$

where $\mathrm{M}$ is related to $\mathrm{j}$ and $\mathrm{k}$ in the manner described in Fig 3 below. 


\begin{tabular}{c|c|c|c|c|c|c}
$j k$ & $x x$ & $y y$ & $z z$ & $y z$ or $z y$ & $x z$ or $z x$ & $x y$ or $y x$ \\
\hline $\mathrm{M}$ & 1 & 2 & 3 & 4 & 5 & 6 \\
Fig 3: Prescription for defining index values of the matrix [d].
\end{tabular}

For the [d] matrix, index $\mathrm{i}$ is also typically written as 1,2 , or 3 . Note that when $\mathrm{j}=\mathrm{k}, \mathrm{d}_{\mathrm{iM}}=\chi_{\mathrm{ijk}}$. This new optical matrix [d] has dimensions $3 \times 6$ and takes the place of the $3 \times 3 \times 3 \chi$ tensor, which allows us to write the $\mathbf{B}_{3}$ in a contracted 2-dimensional matrix notation.

$$
\boldsymbol{B}_{3}=\frac{1}{2} \varepsilon_{0}\left[\begin{array}{llllll}
d_{11} & d_{12} & d_{13} & d_{14} & d_{15} & d_{16} \\
d_{21} & d_{22} & d_{23} & d_{24} & d_{25} & d_{26} \\
d_{31} & d_{32} & d_{33} & d_{34} & d_{35} & d_{36}
\end{array}\right]\left[\begin{array}{c}
E_{1, x} E_{2, x} \\
E_{1, y} E_{2, y} \\
E_{1, z} E_{2, z} \\
E_{1, y} E_{2, z}+E_{1, z} E_{2, y} \\
E_{1, z} E_{2, x}+E_{1, x} E_{2, z} \\
E_{1, x} E_{2, y}+E_{1, y} E_{2, x}
\end{array}\right] .
$$

Each non-linear crystal has different values of $\mathrm{d}_{\mathrm{iM}}$ based upon their chemical and geometrical structures. KDP crystals are tetragonal ${ }^{7}$ crystals of the $42 \mathrm{~m}$ space group. ${ }^{4}$ The optical tensor for KDP in contracted notation is given by expression (21) below. ${ }^{4}$

$$
\left[\begin{array}{cccccc}
0 & 0 & 0 & d_{14} & 0 & 0 \\
0 & 0 & 0 & 0 & d_{14} & 0 \\
0 & 0 & 0 & 0 & 0 & d_{36}
\end{array}\right]
$$

From here, the final form of equation (17) depends upon whether we are studying Type-I or Type-II down-conversion. We shall first focus on Type-I down-conversion, where both the signal and idler photons are ordinary waves and the pump is an extraordinary wave. We shall deal with Type-II afterwards.

Because both the signal and idler are ordinary, $\mathbf{E}_{1}$ and $\mathbf{E}_{2}$ both point in the ordinary direction, and therefore their components will be determined by equation (18).

$$
\begin{aligned}
& E_{1, x}=E_{1} \sin \varphi \\
& E_{2, x}=E_{2} \sin \varphi \\
& E_{1, y}=-E_{1} \cos \varphi \\
& E_{2, y}=-E_{2} \cos \varphi \\
& E_{1, z}=0 \\
& E_{2, z}=0 .
\end{aligned}
$$

Using the optical [d] matrix for the KDP crystal (23), expression (22) allows us to calculate the individual components of $\mathbf{B}_{3}$.

$$
\begin{aligned}
& \mathrm{B}_{3, \mathrm{x}}=0 \\
& \mathrm{~B}_{3, \mathrm{y}}=0 \\
& \mathrm{~B}_{3, \mathrm{z}}=-\frac{1}{2} \varepsilon_{0} \mathrm{E}_{1} \mathrm{E}_{2} \mathrm{~d}_{36} \sin 2 \varphi .
\end{aligned}
$$

The projection of $\mathbf{B}_{3}$ onto the extraordinary direction $\hat{\boldsymbol{e}}$, according to equation (20), is therefore 


$$
B_{3, \|}=\frac{1}{2} \varepsilon_{0} E_{1} E_{2} d_{36} \sin 2 \varphi \sin \theta .
$$

Expression (25) gives us the extraordinary component of $\mathbf{B}_{3}$. This completes the right side of equation (17) for Type-I down-conversion where $\mathrm{n}=3$. We now focus on $\mathbf{A}_{3}$.

\subsection{Coupled Equations for Type-I and Type-II SPDC}

$\mathbf{A}_{3}$ is given by

$$
\mathbf{A}_{3}=\frac{1}{2}\left(-\mathrm{k}_{3}{ }^{2} \mathbf{E}_{3}+\frac{\partial^{2}}{\partial \mathrm{s}^{2}} \mathbf{E}_{3}+2 \mathrm{ik}_{3, \mathrm{~s}} \frac{\mathrm{d}}{\mathrm{ds}} \mathbf{E}_{3}-\mathrm{i}\left(\mathbf{k}_{3} \cdot \frac{\mathrm{d}}{\mathrm{ds}} \mathbf{E}_{3}\right) \hat{\mathbf{s}}+\left(\mathbf{k}_{3} \cdot \mathbf{E}_{3}\right) \mathbf{k}_{3}+\mu \varepsilon \omega_{3}{ }^{2} \mathbf{E}_{3}\right) .
$$

Firstly, $\mathrm{k}^{2}=\mu \varepsilon \omega^{2}$ in matter, so the first and final terms of (26) cancel out. We also make the approximation that the amplitude $\mathrm{E}_{3}$ varies slowly over $\mathrm{s}$, so that the second derivative terms is dwarfed by the first derivative terms, and vanishes.

$$
\left|\frac{\partial^{2}}{\partial s^{2}} E_{3}\right| \ll\left|2 i k_{3, s} \frac{d}{d s} E_{3}\right|
$$

Next, we project (26) onto the extraordinary direction. The term in the $\hat{\boldsymbol{s}}$ direction immediately vanishes because $\hat{\boldsymbol{e}}$ and $\hat{\boldsymbol{s}}$ are orthogonal, while any terms in the $\mathbf{E}_{3}$ direction remain unchanged in magnitude because the pump is assumed to be an extraordinary wave. Given these assumptions, (26) now takes the form

$$
A_{3, \|}=2 i k_{3, s} \frac{d}{d s} E_{3}+k_{3, o}^{2} E_{3} .
$$

Finally, we use the fact that the angle between $\mathbf{E}_{3}$ and $\mathbf{D}_{3}$ (the same angle between $\mathbf{k}_{3}$ and $\hat{\boldsymbol{s}}$ ) is very small for KDP (see Appendix), and also make a low-power approximation for $\mathrm{E}_{3}$, so that the second term of expression (27) becomes much smaller than the first and vanishes. Therefore,

$$
A_{3, \|}=2 i k_{3, s} \frac{d}{d s} E_{3} .
$$

Now replace equation (28) and (25) into equation (17) and solve in terms of $E_{3}$ to attain

$$
\frac{d}{d s} E_{3}=-\frac{i}{4} \frac{\mu \omega_{3}^{2} \varepsilon_{0}}{k_{3, s}} E_{1} E_{2} d_{36} \sin 2 \varphi \sin \theta
$$

If we start again at Section 1.3 with

$$
B_{1, i}=\frac{1}{4} \varepsilon_{0} \chi_{i j k}\left(E_{3, j} E_{2, k}^{*}+E_{3, k} E_{2, j}^{*}\right),
$$

in place of equation (21) and

$$
\boldsymbol{A}_{1}=\frac{1}{2}\left(-k_{1}^{2} \boldsymbol{E}_{1}+\frac{\partial^{2}}{\partial s^{2}} \boldsymbol{E}_{1}+2 i k_{1, s} \frac{d}{d s} \boldsymbol{E}_{3}-i\left(\boldsymbol{k}_{1} \cdot \frac{d}{d s} \boldsymbol{E}_{1}\right) \hat{\boldsymbol{s}}+\left(\boldsymbol{k}_{1} \cdot \boldsymbol{E}_{1}\right) \boldsymbol{k}_{1}+\mu \varepsilon \omega_{1}^{2} \boldsymbol{E}_{1}\right),
$$

in place of equation (26), while repeating the same steps, we attain an equation analogous to (29): 


$$
\frac{d}{d s} E_{1}=-\frac{i}{4} \frac{\mu \omega_{1}^{2} \varepsilon_{0}}{k_{1, s}} E_{3} E_{2}^{*} d_{36} \sin 2 \varphi \sin \theta
$$

Finally, we follow the same steps with

$$
B_{2, i}=\frac{1}{4} \varepsilon_{0} \chi_{i j k}\left(E_{3, j} E_{1, k}^{*}+E_{3, k} E_{1, j}^{*}\right),
$$

in place of equation (21) and

$$
\boldsymbol{A}_{2}=\frac{1}{2}\left(-k_{2}{ }^{2} \boldsymbol{E}_{2}+\frac{\partial^{2}}{\partial s^{2}} \boldsymbol{E}_{2}+2 i k_{2, s} \frac{d}{d s} \boldsymbol{E}_{2}-i\left(\boldsymbol{k}_{2} \cdot \frac{d}{d s} \boldsymbol{E}_{2}\right) \hat{\boldsymbol{s}}+\left(\boldsymbol{k}_{2} \cdot \boldsymbol{E}_{2}\right) \boldsymbol{k}_{2}+\mu \varepsilon \omega_{2}{ }^{2} \boldsymbol{E}_{2}\right),
$$

in place of (26) to achieve a third equation:

$$
\frac{d}{d s} E_{2}=-\frac{i}{4} \frac{\mu \omega_{2}^{2} \varepsilon_{0}}{k_{3,2}} E_{3} E_{1}^{*} d_{36} \sin 2 \varphi \sin \theta
$$

Equations (29), (30), and (31) show the coupled nature of the three plane waves involved in Type-I down-conversion from a KDP crystal. Similar equations are found in several textbooks on the subject of non-linear optics. $4,8,9$

Type-II down-conversion has similar coupled equations. The difference is that one of the outgoing photons is an extraordinary wave. To find the coupled equations for Type-II SPDC, the components of the electric fields in the matrix equation (22) are determined by using equation (18) for one of the waves, say $\mathbf{E}_{1}$, and equation (19) for the other, say $\mathbf{E}_{2}$.

$$
\begin{aligned}
& E_{1, x}=E_{1} \sin \varphi \\
& E_{2, x}=E_{2} \cos \theta \cos \varphi \\
& E_{1, y}=-E_{1} \cos \varphi \\
& E_{2, y}=E_{2} \cos \theta \sin \varphi \\
& E_{1, z}=0 \\
& E_{2, z}=-E_{2} \sin \theta .
\end{aligned}
$$

Using these components and matrix (23) in equation (22), the components of $\mathbf{B}_{3}$ for Type-II are

$$
\begin{aligned}
B_{3, x} & =\frac{1}{2} \varepsilon_{0} E_{1} E_{2} d_{14} \cos \varphi \sin \theta \\
B_{3, y} & =-\frac{1}{2} \varepsilon_{0} E_{1} E_{2} d_{14} \sin \varphi \sin \theta \\
B_{3, y} & =\frac{1}{2} \varepsilon_{0} E_{1} E_{2} d_{36} \cos \theta\left(\sin \varphi^{2}-\cos \varphi^{2}\right) .
\end{aligned}
$$

To take the projection of $\mathbf{B}_{3}$ along $\hat{\boldsymbol{e}}$, replace (32) into equation (20).

$$
B_{3, \|}=\frac{1}{2} \varepsilon_{0} E_{1} E_{2}\left(d_{14}+d_{36}\right)\left(\cos \varphi^{2}-\sin \varphi^{2}\right) \sin \theta \cos \theta
$$

From equation (33), the derivation is identical to Type-I. The coupled equations for Type-II SPDC are 


$$
\begin{aligned}
& \frac{d}{d s} E_{3}=-\frac{i}{4} \frac{\mu \omega_{3}^{2} \varepsilon_{0}}{k_{3, s}} E_{1} E_{2}\left(d_{14}+d_{36}\right)\left(\cos \varphi^{2}-\sin \varphi^{2}\right) \sin \theta \cos \theta \\
& \frac{d}{d s} E_{1}=-\frac{i}{4} \frac{\mu \omega_{1}^{2} \varepsilon_{0}}{k_{1, s}} E_{3} E_{2}{ }^{*}\left(d_{14}+d_{36}\right)\left(\cos \varphi^{2}-\sin \varphi^{2}\right) \sin \theta \cos \theta \\
& \frac{d}{d s} E_{2}=-\frac{i}{4} \frac{\mu \omega_{2}^{2} \varepsilon_{0}}{k_{2, s}} E_{3} E_{1}{ }^{*}\left(d_{14}+d_{36}\right)\left(\cos \varphi^{2}-\sin \varphi^{2}\right) \sin \theta \cos \theta
\end{aligned}
$$

The only difference between the Type-I coupled equations (29), (30), and (31) and the Type-II coupled equations (34), (35), and (36) is the last constant containing components of the [d] matrix and polar coordinates. These factors can be condensed into a single constant, $\mathrm{d}_{\mathrm{eff}}$. For Type-I SPDC,

$$
d_{e f f}=d_{36} \sin 2 \varphi \sin \theta
$$

For Type-II,

$$
d_{e f f}=\left(d_{14}+d_{36}\right)\left(\cos \varphi^{2}-\sin \varphi^{2}\right) \sin \theta \cos \theta
$$

\subsection{Polarization Entanglement for Type-I and Type-II SPDC}

Now that a theory has been developed for the creation of polarization-correlated photons using a single frequency laser (the pump) and a uniaxial crystal, we analyze entanglement of the polarization states of the signal and idler. This chapter will describe methods for attaining polarization entanglement for both Type-I and Type-II SPDC.

Quantum mechanics is a fundamentally statistical theory. This means that it does not attempt to predict what will happen, only the probabilities of certain events. Mathematically, a system that could be in one of multiple states is represented as a linear combination of those states. For example, if the polarization of a photon is going to be measured, and can either be polarized horizontally or vertically, the state of the photon, $\psi$, is given by

$$
|\psi\rangle=a|H\rangle+b|V\rangle
$$

where $\mathrm{a}$ and $\mathrm{b}$ are complex numbers which give weight to $|H\rangle$, the horizontal state, and $|V\rangle$, the vertical state, based upon their probabilities, such that $|a|^{2}+|b|^{2}=1$. The photon is said to be in a superposition of horizontal and vertical polarization if neither a nor $b$ is equal to zero.

Quantum entanglement between two particles occurs when the particles are in a quantum superposition of states, and their states are correlated. Both types of SPDC have correlated photon polarization. In Type-II down-conversion, photons can be found in a superposition of polarization states where the signal and idler cones intersect (Fig 4). On one cone lie extraordinarily polarized rays, while the other has ordinary rays. At the intersection points, it cannot be determined from which cone a photon originated, and therefore it cannot be determined what the polarization of a photon is before a measurement is taken. This means a photon at an intersection point is in a superposition of ordinary and extraordinary (or horizontal and vertical) polarization states. Because the photons produced also have correlated polarizations, the photons found at the intersection points are entangled. The state of this system is given by

$$
\left|\psi_{2}\right\rangle=a|H\rangle_{A}|V\rangle_{B}+b|V\rangle_{A}|H\rangle_{B},
$$




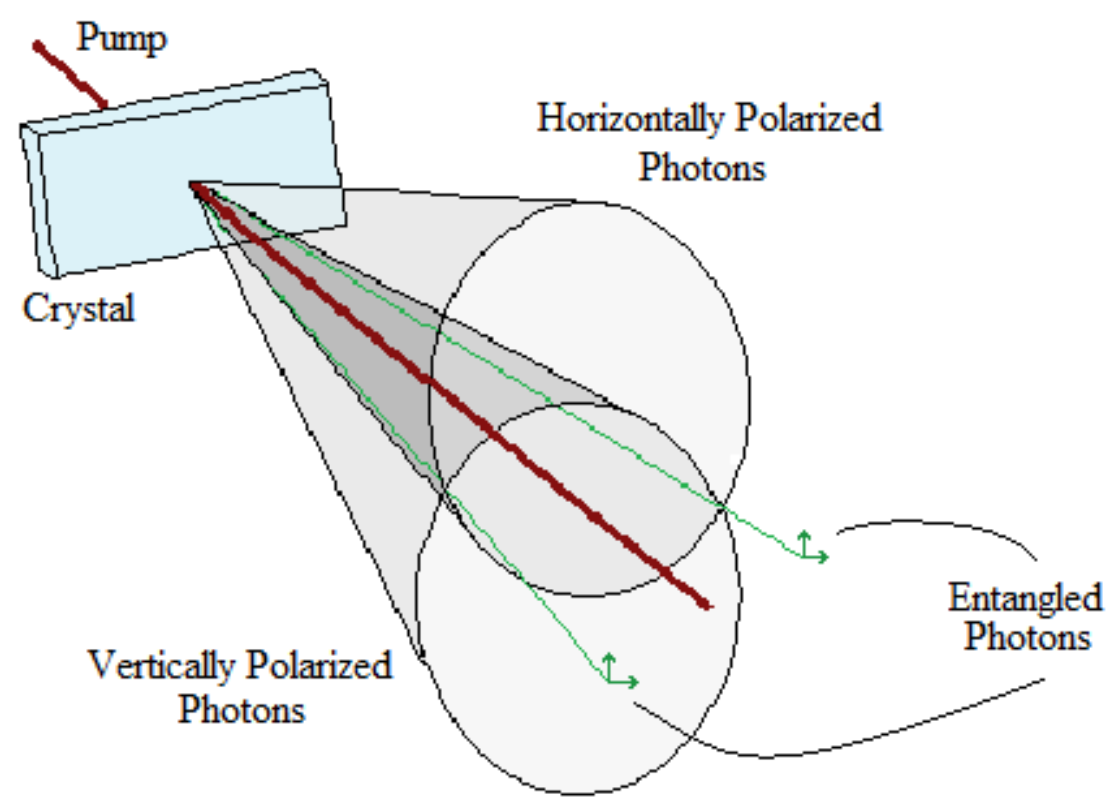

Fig 4: Type-II entangled photons from SPDC crystal. When a photon from one cone has horizontal polarization, another photon exists on the other cone with vertical polarization. This is correlation. When a photon exists on an intersection point, it cannot be said from which cone it came from, and it is thus in a superposition of polarization states. The combination of correlation and superposition leads to entanglement.

where subscripts A and B refer to photon A and photon B.

In Type-I SPDC, the signal and idler are both ordinarily polarized, so although their polarizations are correlated, neither are in a superposition of states. Entangled photons can still be produced from Type-I down-conversion, but it requires the use of two crystals with identical optical axes. When the crystals are placed back to back, their axes must be orientated such that one is rotated about the direction of pump propagation by $90^{\circ}$ from the other. Using the two crystal axis directions as a basis, the pump will be seen as being in a superposition of ordinary and extraordinary polarizations until it interacts with one of the crystals. SPDC may occur if one of the crystals "observes" an extraordinary pump. As long as the pump beam was not polarized exactly along one of the crystal axes, it will be unknown through which crystal the pump beam was down-converted (see Fig 5). The crystals must be thin enough so that the cones cannot be distinguished at the detection zone, usually about 1 $\mathrm{mm}$. For more on the effects of crystal length on down-conversion, see Ramirez et. al. ${ }^{10}$

If two photons, A and B, are entangled through Type-I down-conversion and measured in the basis of horizontal polarization $|\mathrm{H}\rangle$ and vertical polarization $|\mathrm{V}\rangle$, then the state of the system is written as

An entangled state is said to be maximally entangled if $a=b$. In this case, the state weighted by coefficient $a$ is just as likely to be measured as the state weighted by coefficient $b$. This maximally entangled pair is called a Bell state. For Type-I entanglement, this can be achieved by setting the incoming photon to have polarization at a $45^{\circ}$ angle relative to both crystal axes. Polarizations entangled photons are always produced in a Bell state in Type-II SPDC because the signal and idler will always have opposite polarizations. 


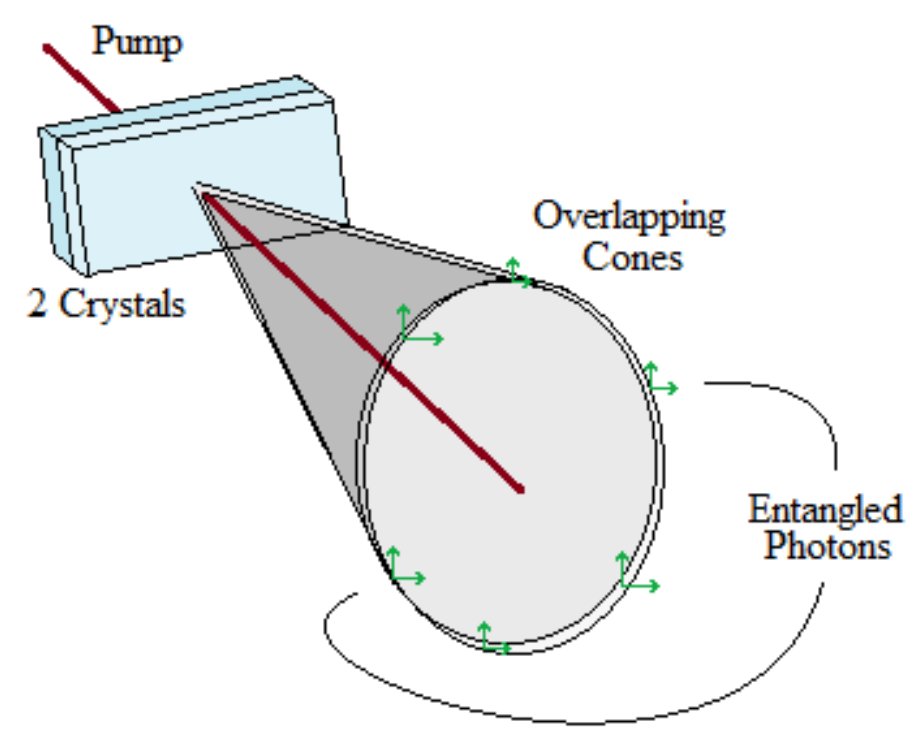

Fig 5: Type-I entangled photons from two identical uniaxial crystals. The crystal axis of the $2^{\text {nd }}$ crystal is rotated $90^{\circ}$ about the pump propagation direction from the $1^{\text {st }}$ crystal axis. If SPDC occurs in crystal \#1, both outgoing photons will be ordinarily polarized relative to the $1^{\text {st }}$ crystal axis. If SPDC occurs in crystal \#2, they will be ordinary to the $2^{\text {nd }}$ axis. This is correlation. The cones are too close together to determine from which crystal the photons originate, so they are in a superposition of polarization states. Entangled pairs can be found at any points around the cone, opposite each other.

The four Bell states for a pair of photon entangled particles are

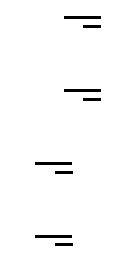

The states are only possible from Type-I down-conversion, while the states are only possible from Type-II down-conversion. Bell states are typically preferable in entanglement experiments, as all possible results of a measurement are equally likely to occur.

\subsection{Bell Inequality and Local-realism}

Entanglement, like the superposition of states, is a quantum mechanical effect that is completely foreign to our classical intuition. For example, when an electron-positron pair is created from the decay of a spin-0 particle, they will have opposite spins. When their spins are measured in the same basis, the results of these measurements will always be opposites. This means that if the spin of the electron is measured, the result of a similar spin measurement on the positron will be immediately known to the observer, even if the positron has yet to be measured.

Quantum effects are known to break fundamental assumptions we make about the nature of the universe. One such assumption is known as realism, which is the idea that particles exist in definite states before being observed. In other words, if a tree falls in a forest and no one is around to hear it, a realist would say that it does make a sound. Another idea we hold about the universe is that no information can be transmitted faster than the speed of light (locality), though this idea is backed by much observation. Together, these ideas are known as local-realism. Superposition of states and 
entanglement, which are not part of our classical world view, create problems for us under these assumptions.

For some time, the apparent randomness of quantum mechanics was thought to be due to unknown effects that were not taken into account by quantum theory. The Bell Inequality was the first rigorous mathematical theory to both challenge our perception of local-realism and hidden variables in a testable and predictable manner. Bell's theorem invoked the use of expectation values.

When photons are produced via Type-II down-conversion, they are always measured to have opposite polarization when measured using similarly oriented polarizers. If there is a local hidden variable, say $\lambda$, which hides a deterministic explanation of quantum theory, then there must be functions of $\lambda$ and polarizer direction which give the polarizations of photons $\mathrm{A}$ and $\mathrm{B}$. Let these functions be defined as $A(\vec{a}, \lambda)$ and $B(\vec{b}, \lambda)$ respectively. Let horizontal polarization be given a value of +1 , and vertical polarization be given -1 . Therefore, these functions are limited to:

$$
A(\vec{a}, \lambda)= \pm 1
$$

and

$$
B(\vec{b}, \lambda)= \pm 1
$$

Because these photons always have opposite polarization, if they are measured in the same direction $\vec{a}$,

$$
A(\vec{a}, \lambda)=-B(\vec{a}, \lambda)
$$

If $\mathrm{p}(\vec{a}, \vec{b})$ is the expectation value of the products of $A(\vec{a}, \lambda)$ and $B(\vec{b}, \lambda)$, then

$$
p(\vec{a}, \vec{b})=\int \rho(\lambda) A(\vec{a}, \lambda) B(\vec{b}, \lambda) d \lambda
$$

where $\rho(\lambda)$ is the probability density for the hidden variable, supposing it exists. Using equation (39), $B(\vec{b}, \lambda)$ can be replaced with $-A(\vec{b}, \lambda)$. If we introduce another polarizer direction using the unit vector $\vec{c}$, we can find a difference value between two different expectation values.

$$
p(\vec{a}, \vec{b})-p(\vec{a}, \vec{c})=-\int \rho(\lambda)[A(\vec{a}, \lambda) A(\vec{b}, \lambda)-A(\vec{a}, \lambda) A(\vec{c}, \lambda)] d \lambda .
$$

Given that the hidden variable function can only result in we see \pm 1 for any polarizer direction, we see that $[A(\vec{b}, \lambda)]^{2}=1$. Therefore we are safe to multiply by $[A(\vec{b}, \lambda)]^{2}$ without changing any values.

$$
p(\vec{a}, \vec{b})-p(\vec{a}, \vec{c})=-\int \rho(\lambda)[A(\vec{a}, \lambda) A(\vec{b}, \lambda)-A(\vec{a}, \lambda) A(\vec{c}, \lambda)][A(\vec{b}, \lambda)]^{2} d \lambda .
$$

We can now rearrange equation (42) by factoring out $A(\vec{a}, \lambda)$ and distributing $A(\vec{b}, \lambda)$.

$$
p(\vec{a}, \vec{b})-p(\vec{a}, \vec{c})=-\int \rho(\lambda)[1-A(\vec{b}, \lambda) A(\vec{c}, \lambda)] A(\vec{a}, \lambda) A(\vec{b}, \lambda) d \lambda .
$$

From equation (38a), we see that $A(\vec{a}, \lambda) A(\vec{b}, \lambda)= \pm 1$. This is also true of $A(\vec{b}, \lambda) A(\vec{c}, \lambda)$. Because $\rho(\lambda)$ is a positive number, it is also true that $\rho(\lambda)[1-A(\vec{b}, \lambda) A(\vec{c}, \lambda)] \geq 0$. Therefore, if we take the absolute value of $\mathrm{p}(\vec{a}, \vec{b})-\mathrm{p}(\vec{a}, \vec{c})$, 


$$
|p(\vec{a}, \vec{b})-p(\vec{a}, \vec{c})| \leq \int \rho(\lambda)[1-A(\vec{b}, \lambda) A(\vec{c}, \lambda)] d \lambda .
$$

Substituting $B(\vec{c}, \lambda)$ for $-A(\vec{c}, \lambda)$, and taking the integration in equation (44), we end up with the Bell Inequality.

$$
|p(\vec{a}, \vec{b})-p(\vec{a}, \vec{c})| \leq 1+p(\vec{b}, \vec{c}) .
$$

This inequality was developed in this form in $1964^{11}$ by J.S. Bell under the assumptions that the hidden variable was local (in that information does not travel faster than light) and that the measurable quantities exist regardless of whether or not a measurement was taken (realism). A violation of this inequality is therefore a violation of one or both of these assumptions. The same exact inequality can be derived for Type-I down-conversion as well. The only difference in the derivation is that in Type-I, polarizations are always the same if measured in the same way, so equation (39) becomes $A(\vec{a}, \lambda)=$ $B(\vec{a}, \lambda)$.

After the development of equation (45), a more intuitive formulation was built by Hardy ${ }^{12}$ upon the same basic assumptions. Instead of using the expectation value of the product of the measurement results, Hardy simply used the probabilities of various states.

The state of a photon of unknown polarization can be written as a function of the angle $\theta$ relative to the horizontal direction.

$$
|\theta\rangle=\cos \theta|H\rangle+\sin \theta|V\rangle .
$$

The probability to detect photons entangled by Type-I down-conversion in state $\left(\theta_{\mathrm{Ai}}, \theta_{\mathrm{Bj}}\right)$ can now be found. By taking the squared absolute value of the dot product of the initial entangled state $\mathrm{Eq}(3)$ with the final state ${ }_{A}\left\langle\left.\theta_{\mathrm{i}}\right|_{\mathrm{B}}\left\langle\theta_{\mathrm{j}}\right|\right.$, the probability is found to be

$$
\begin{aligned}
& P\left(\theta_{A i}, \theta_{B j}\right)=\mid\left\langle\left.\left.\theta_{A i}\right|_{A}\left\langle\left.\theta_{B j}\right|_{B} \mid \psi_{1}\right\rangle\right|^{2}\right. \\
& \quad=\left|\left(\operatorname { c o s } \theta _ { A i } \left\langleH | + \operatorname { s i n } \theta _ { A i } \langle V | ) \left(\operatorname { c o s } \theta _ { B j } \left\langle\left.H\left|+\sin \theta_{B j}\langle V|\right)\left(a|H\rangle_{A}|H\rangle_{B}+b|V\rangle_{A}|V\rangle_{B}\right)\right|^{2}\right.\right.\right.\right.\right.
\end{aligned}
$$

Simplifying equation (57) we attain

$$
P\left(\theta_{A i}, \theta_{B j}\right)=\left|a \cos \theta_{A i} \cos \theta_{B j}+b \sin \theta_{A i} \sin \theta_{B j}\right|^{2} .
$$

Hardys Inequality entangled photons is

$$
P\left(\theta_{A 2}, \theta_{B 2}\right) \geq P\left(\theta_{A 1}, \theta_{B 1}\right)-P\left(\theta_{A 2} \pm 90^{\circ}, \theta_{B 1}\right)-P\left(\theta_{A 1}, \theta_{B 2} \pm 90^{\circ}\right) .
$$

Though the Hardy Test can be used for any general quantum entanglement, in this paper, we apply it only to down-conversion and polarization states. This expression is derived more rigorously by Mermin $^{13}$. Expression (59) inhibits $\mathrm{P}\left(\theta_{\mathrm{A} 2}, \theta_{\mathrm{B} 2}\right)$ from going below a certain threshold if local-realism is to stand. Each individual term of this inequality is experimentally determined using the number of coincident detections of photons in state $\left(\theta_{\mathrm{Ai}}, \theta_{\mathrm{Bj}}\right)$, given by $\mathrm{N}\left(\theta_{\mathrm{Ai}}, \theta_{\mathrm{Bj}}\right)$.

$$
P\left(\theta_{A i}, \theta_{B j}\right)=\frac{N\left(\theta_{A i}, \theta_{B j}\right)}{N\left(\theta_{A i}, \theta_{B j}\right)+N\left(\theta_{A i} \pm 90^{\circ}, \theta_{B j}\right)+N\left(\theta_{A i}, \theta_{B j} \pm 90^{\circ}\right)+N\left(\theta_{A i} \pm 90^{\circ}, \theta_{B j} \pm 90^{\circ}\right)} .
$$

In order to better understand the Hardys inequality, consider this thought experiment. Imagine a 
single photon source that produces pairs of entangled photons that are sent in different directions, such as a down-conversion crystal. One photon goes to detector A, and is observed by Alice, while the other goes to $\mathrm{B}$ and is observed by Bob. Before reaching their respective detector, the photons must pass through a respective polarizer. Polarizer $A$ is oriented arbitrarily to either angle $\theta_{\mathrm{A} 1}$ or $\theta_{\mathrm{A} 2}$ just after the photons are produced and just before measurement. Polarizer B is similarly randomly oriented to either $\theta_{\mathrm{B} 1}$ or $\theta_{\mathrm{B} 2}$. This constraint is to make sure no information about one polarizer has time to reach the other before a measurement is taken.

In this experiment, the probability for both Alice to detect a photon with polarization $\theta_{\mathrm{Ai}}$ and Bob to detect a photon with polarization $\theta_{\mathrm{Bj}}$ is $\mathrm{P}\left(\theta_{\mathrm{Ai}}, \theta_{\mathrm{Bj}}\right)$. The probability of Alice to detect a photon with polarization $\theta_{\mathrm{A} 1}$ given Bob detected a photon at $\theta_{\mathrm{Bj}}$ is $\mathrm{P}\left(\theta_{\mathrm{Ai}} \mid \theta_{\mathrm{Bj}}\right)$.

After many trials are run, assume that Alice and Bob exchange and compare their data, and the following observations are made:

1 If detector $\mathrm{A}$ is set to $\theta_{\mathrm{A} 1}$ and detector $\mathrm{B}$ is set to $\theta_{\mathrm{B} 1}$, there is a nonzero probability for both Alice and Bob to detect a photon at their counter.

$$
P\left(\theta_{A 1}, \theta_{B 1}\right)>0
$$

2 Given that Bob has detected a photon with polarizer $\theta_{\mathrm{B} 1}$, Alice always detects a photon at angle $\theta_{\mathrm{A} 2}$

$$
P\left(\theta_{A 2} \mid \theta_{B 1}\right)=1
$$

3 Given that Alice has detected a photon with polarizer $\theta_{\mathrm{A} 1}$, Bob always detects a photon at angle $\theta_{\mathrm{B} 2}$

$$
P\left(\theta_{B 2} \mid \theta_{A 1}\right)=1
$$

Applying our classical logic to these three observation, we can extrapolate what should logically be observed regarding $\mathrm{P}\left(\theta_{\mathrm{A} 2}, \theta_{\mathrm{B} 2}\right)$. As stated before, we shall assume that polarization states exist regardless of being measured. Given observation 2 and 3, whenever Bob detects a photon with polarization $\theta_{\mathrm{B} 1}$ and Alice detects a photon with polarization $\theta_{\mathrm{A} 1}$, had they instead chosen to orientate their polarizers at $\theta_{\mathrm{B} 2}$ and $\theta_{\mathrm{A} 2}$, they would be at least as likely to detect a photon at those positions as well.

$$
P\left(\theta_{A 2}, \theta_{B 2}\right) \geq P\left(\theta_{A 1}, \theta_{B 1}\right)
$$

This relationship does not take into account the probabilistic nature of the measurements. It could be that $\mathrm{P}\left(\theta_{\mathrm{A} 2} \mid \theta_{\mathrm{B} 1}\right)$ and $\mathrm{P}\left(\theta_{\mathrm{B} 2} \mid \theta_{\mathrm{A} 1}\right)$ are slightly less than 1 , but the coincidental measurements simply happened to be made every time. We account for this possibility by restating observations 2 and 3 in a logically equivalent manner and then examining the effect of experimental error. According to observation 2, if Bob's photon is found polarized along $\theta_{\mathrm{B} 1}$, then Alice always find her photon with polarization $\theta_{\mathrm{A} 2}$. This means that it would never be the case that Alice finds her photon along $\theta_{\mathrm{A} 2} \pm 90^{\circ}$ and Bob finds his along $\theta_{\mathrm{B} 1}$.

$$
P\left(\theta_{A 2} \mid \theta_{B 1}\right)=1 \rightarrow P\left(\theta_{A 2} \pm 90^{\circ}, \theta_{B 1}\right)=0
$$

Similarly, according to observation 3, Bob will never find his photon along $\theta_{\mathrm{B} 2} \pm 90^{\circ}$ if Alice's is along $\theta_{\mathrm{A} 1}$.

$$
P\left(\theta_{B 2} \mid \theta_{A 1}\right)=1 \rightarrow P\left(\theta_{A 1}, \theta_{B 2} \pm 90^{\circ}\right)=0
$$

We must recognize that it is impossible to experimentally prove that any event has zero likelihood. Any seemingly impossible event may not occur after millions of trials, and yet it may occur on the next run. If $\mathrm{P}\left(\theta_{\mathrm{A} 2} \pm 90^{\circ}, \theta_{\mathrm{B} 1}\right)$ and $\mathrm{P}\left(\theta_{\mathrm{A} 1}, \theta_{\mathrm{B} 2} \pm 90^{\circ}\right)$ was nonzero, say 0.01 each, then there would be a reduction of 0.02 from $\mathrm{P}\left(\theta_{\mathrm{A} 2}, \theta_{\mathrm{B} 2}\right)$. Given this possibility, expression (61) must be modified. 
This is the Hardy inequality from expression (59). Finally, after calculating each probability using their experimental data, assume that Alice and Bob compare notes regarding $\theta_{\mathrm{A} 2}$ and $\theta_{\mathrm{B} 2}$, and they find that the two measurements never coincide.

This result would lead to a violation of the Hardy inequality, which would mean a violation of our classical intuition. Indeed, real world examples of a violation of expression (59) have been verified for down-conversion and other quantum entanglement phenomena. ${ }^{14}$

\subsection{Quantum Teleportation using Entangled Photons}

It has long been established that any measurement of a particle in a superposition of states replaces the probability distribution with a definitive state. Based upon the Heisenberg uncertainty principle, we also know that it is also impossible to know all quantities related to a particle simultaneously. Given these facts, it seems impossible to scan a group of particles, and reconstruct them exactly as they were in another location. This makes it seem that even non-instantaneous teleportation is impossible, but the answer to this problem is found in entanglement.

We start with a source of polarization entangled photons A and B. Let them be entangled by Type-I down-conversion, so that they will either both be horizontal or both be vertical when measured using similarly oriented polarizers. Make sure that the entangled pair is in a Bell state, so that the photons are just as likely to be horizontally or vertically polarized relative to the pump. By entangling another photon (let's call it photon X) with photon A and measuring the type of entanglement that A and $\mathrm{X}$ share, we can then send that information to $\mathrm{B}$ through a classical signal and change $\mathrm{B}$ so that it has the same polarization as $\mathrm{X}$, all without ever knowing their polarizations (see Fig 6).

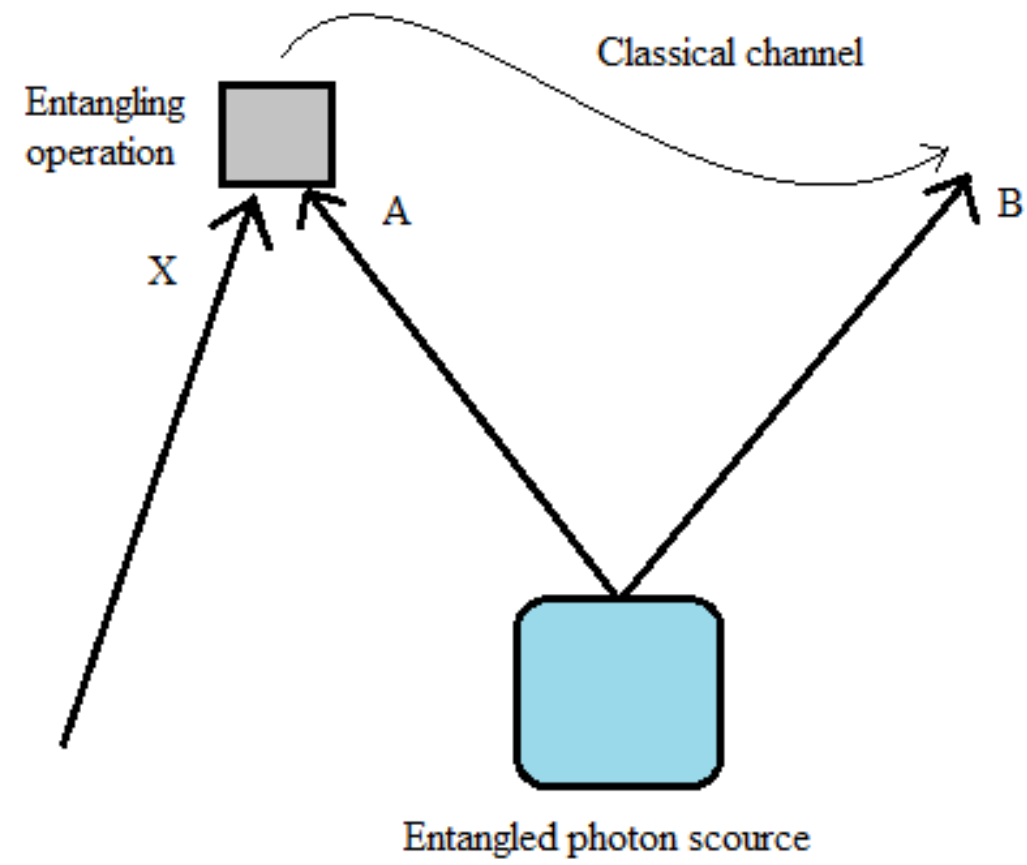

Fig 6: Basic diagram for quantum teleportation of photon X. Photons A and B are entangled, usually through SPDC, and then photons $\mathrm{A}$ and $\mathrm{X}$ are also entangled. Information about the nature of the entanglement is sent to photon $\mathrm{B}$. 
The method of entangling photons A and X can be very difficult, and requires careful timing and precision. In general however, A and X can interact with a polarizing beam splitter simultaneously, and through that interaction become entangled in their polarization (see Fig 7). Following the beam splitter, two detectors can be placed in the paths of the photons (see Fig 8). If photons A and X have are polarized such that their polarizations will always be measured as orthogonal, then both detectors will click. If the photons are entangled such that they have the same polarization, only one of the detectors will click. Photon X could be produced using the same crystals to make sure that their polarizations are always either aligned or orthogonal. This shows that different Bell states can produce different effects.

If two clicks are detected, rotating B by $90^{\circ}$ with a polarization rotating crystal that can be controlled externally will make B have the same polarization as X. Likewise, if one click is detected, B is already the same as X. Although photons A and X must be destroyed in this process, the quantum state of $\mathrm{X}$ is transferred to $\mathrm{B}$ without ever having to measure it in a way that would give us unique information of its polarization. All that is measured is which kind of entanglement is shared between photons $\mathrm{A}$ and $\mathrm{X}$. This information would have to be sent through the classical channel before photon $\mathrm{B}$ reached its destination. Information through the classical channel cannot travel faster than light, so photon B must be delayed in some way. This could be achieved by lengthening its path using mirrors, or by sending it through a medium which significantly slows its speed. Quantum teleportation could also be achieved through Type-II down-conversion if B is kept unchanged when two clicks are detected, and instead rotate B if one click is detected.
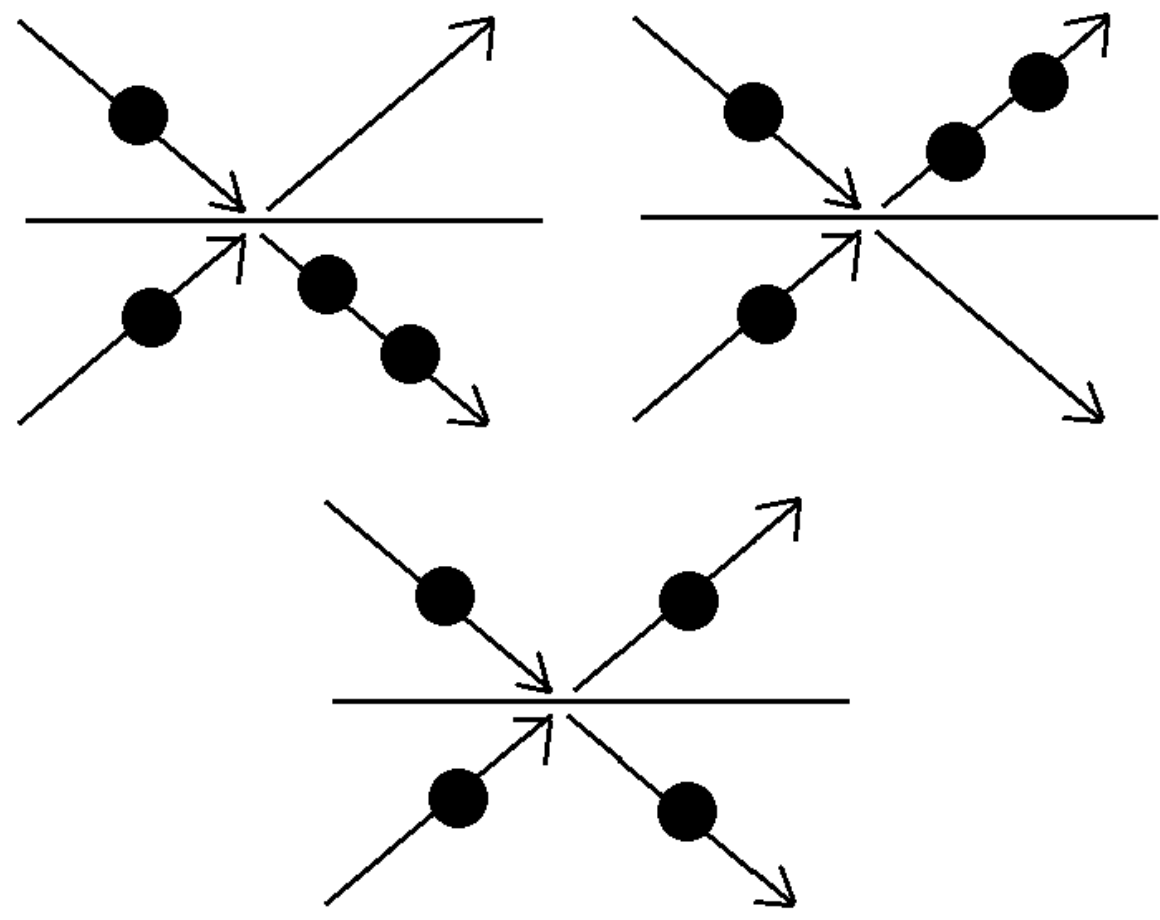

Fig 7: Entangled photons interacting with a polarizing beam splitter simultaneously. TOP: When two photons are entangled such that they always have the same polarization, their probability waves will interfere such that they will only be detected on the same side of the beam splitter after interacting with it. BOTTOM: When polarized orthogonally, the photons will always exit on opposite sides of the beam splitter. It is impossible to tell whether they were reflected or if they passed through. 


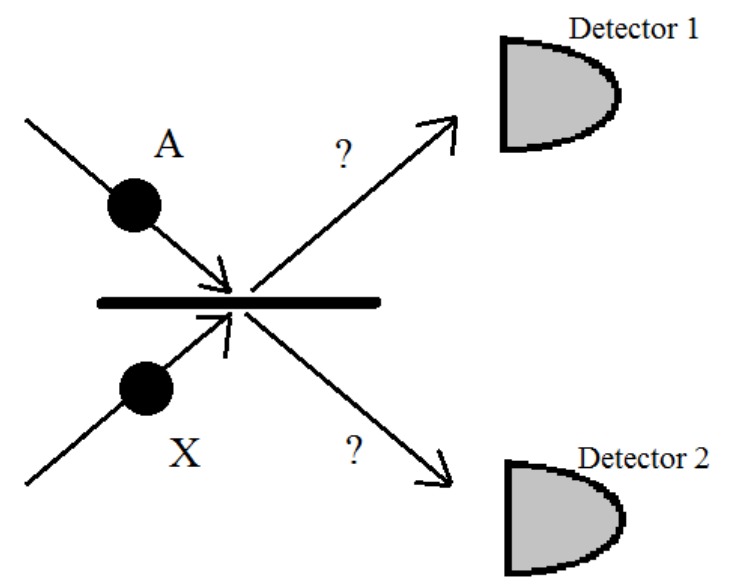

Fig 8: Photons $\mathrm{A}$ and $\mathrm{X}$ are sent to a beam splitter simultaneously and are entangled once exiting. The outgoing photons will be detected by either Detector 1, Detector 2, or both depending upon the entanglement relationship between the two photons. The result will not tell us the state of either photon, but it will give us the entanglement state.

\subsection{Entanglement Transfer from Photons to Matter}

Now let us examine what happens when photon $\mathrm{X}$ is entangled with another photon before being entangled with photon $\mathrm{A}$. If $\mathrm{X}$ is entangled with $\mathrm{A}$ without measuring their individual states, as was done in section 3.1, then the information of photon $\mathrm{X}$ will be transferred to photon $\mathrm{B}$. However, if $\mathrm{X}$ is entangled with photon $\mathrm{Y}$, as in Fig 9, the only information that is known about $\mathrm{X}$ is that it is in superposition of polarization states and is correlated to $\mathrm{Y}$. That is exactly the information that is transferred to B. Similarly, the only information known about A is that it is entangled with B, therefore that information is transferred to $\mathrm{Y}$. The result is that photons B and $\mathrm{Y}$ are now entangled, not through interaction with each other, but through the interactions of $\mathrm{A}$ and $\mathrm{X}$. This phenomenon is called entanglement transfer. ${ }^{1}$

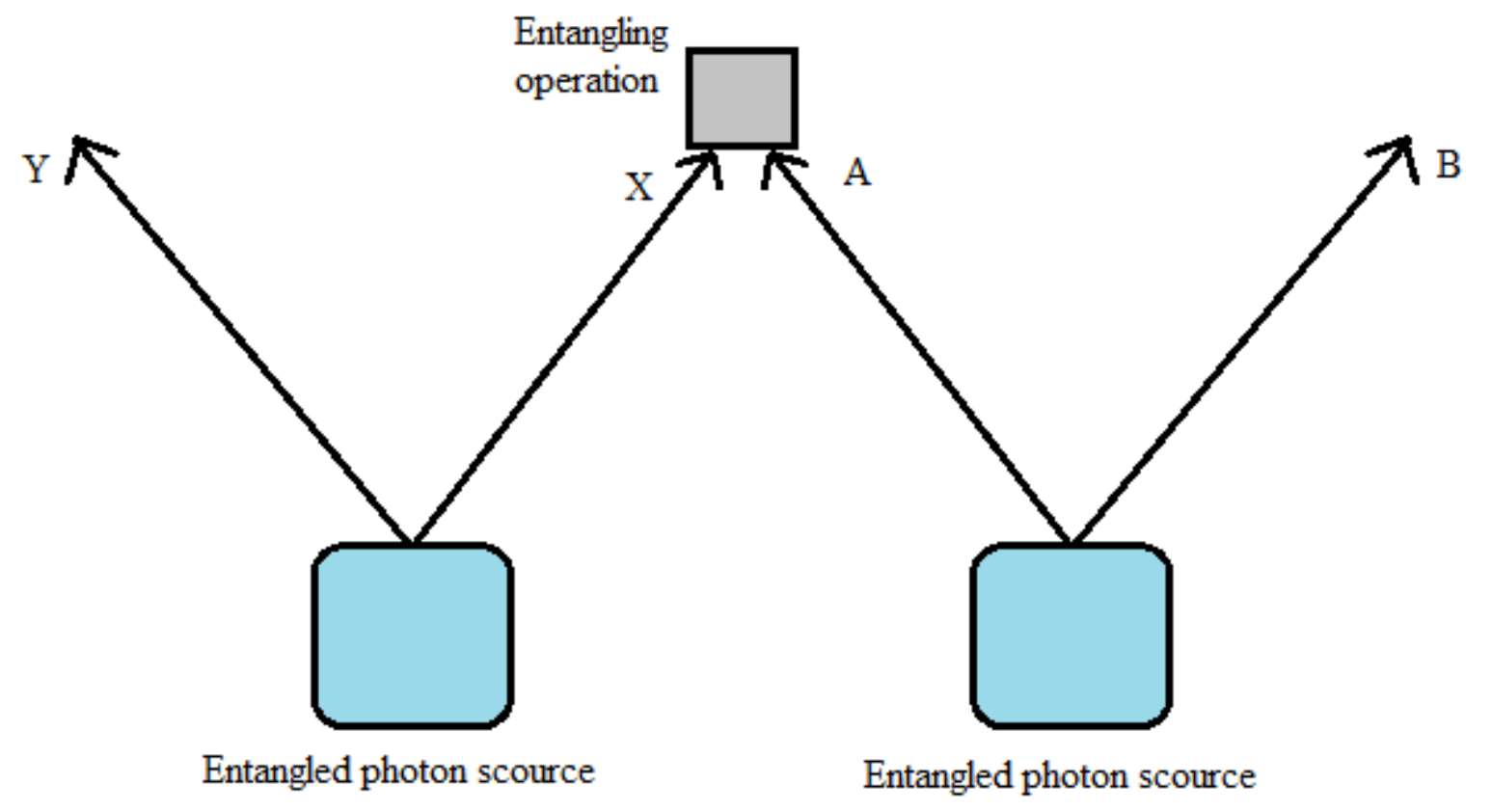

Fig 9: Diagram for entanglement transfer from photons $A$ and $X$ to photons $B$ and $Y$. Photons A and B are initially entangled, as are photons $\mathrm{Y}$ and $\mathrm{X}$. Photons $\mathrm{A}$ and $\mathrm{X}$ are then entangled. Information about the nature of the entanglement is transferred to photons B and $\mathrm{Y}$. 
Entanglement transfer need not occur exclusively between photons. It has been observed that entangled photons can interact with plasmons on periodically perforated metal plates, be reradiated on the other side of their respective plates, and still retain their entanglement (see Fig 10). ${ }^{15}$ Plasmons are localized electromagnetic waves formed at a dielectric-metal interface, which are stimulated by interactions with light, usually heavily dependent on frequency. The propagation of plasmons are associated with the collective motion of a large number of electrons. The perforations on the metal plates are periodically spaced and smaller than the wavelength of the incident light, which leads to a very efficient light transmission where light tunnels through each sub-wavelength aperture. This is an indication of a cooperative effect where incident photons are scattered off the periodic arrangement of holes and converted into a surface-plasmon. In the case of plasmons incidnt down-converted photons, we expect them to pass through the holes to reradiate on the other side of the plate. If an incoming photon is horizontally polarized, the electron wave will transfer energy horizontally across the metal through horizontal oscillations. When the plasmons pass through the perforated surface, the direction of oscillation remains the same. Finally, when the plasmons reradiate another photon, these horizontal oscillations in the material will produce a horizontally polarized photon. Because entanglement can easily be destroyed through interactions, and given the collective nature of the surface plasmons, which involves large numbers of electrons, it seems remarkable that entanglement survives. It can be inferred that the plasmons on the metal plates retained the entanglement during transition from light to matter. The entanglement is transferred because the direction of polarization for either individual photon is unknown, so it is unknown which plate is experiencing horizontal oscillations, and which is experiencing vertical oscillations.

One application for entanglement has been in quantum computing, where the classical two-state bit is replaced with the qubit, which can be in multiple states simultaneously. By entangling multiple qubits, quantum computers are theorized to be able to excel in parallel computing where classical computers cannot compare. The main limitation has been in both creating many entangled states and storing them so that their entanglement is stable. While it is easy to entangle a number of photons, it is very difficult to store them for future use. Also, while even single particles can be contained and arranged, such as charged particles within ion traps ${ }^{16}$, it can be difficult to arrange and manipulate them after they have been entangled. Transferring entanglement from light to matter could potentially ease these difficulties, as it combines the simplicity of entangling photons with SPDC with the ease of storage of particles.

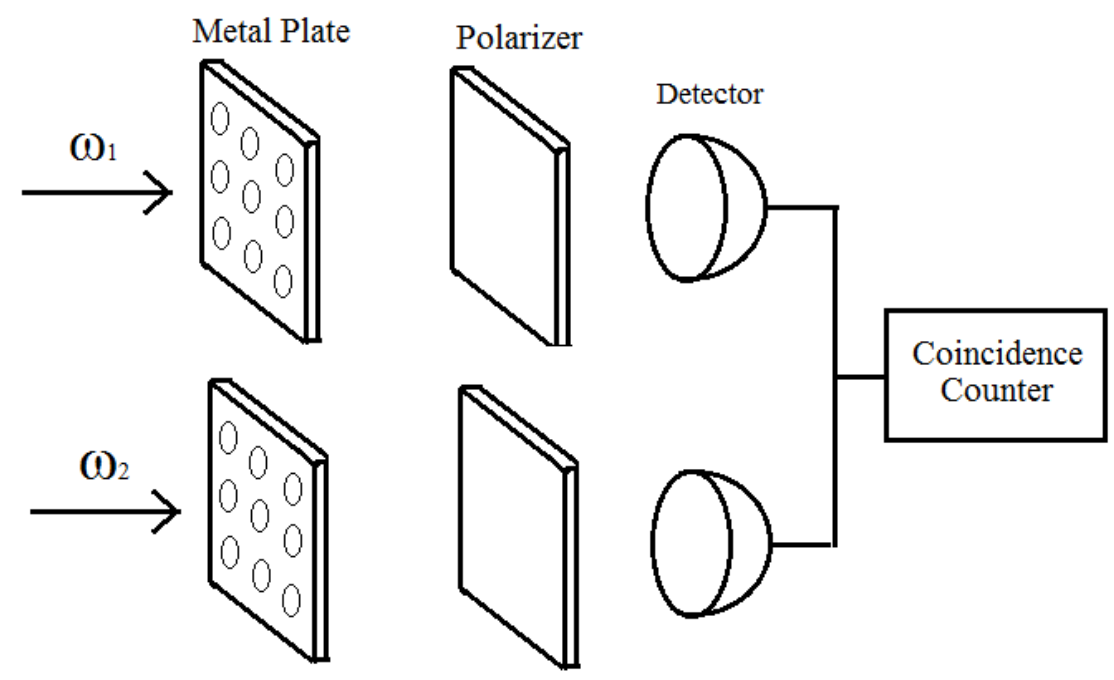

Fig 10: Schematic for photon-to-plasmon entanglement transfer. Down-converted photons stimulate plasmons on metal plates with regular perforations. Reradiated photons pass through identical polarizers then to photon detectors. The entanglement is verified using the coincidence counter. 


\section{Conclusion}

A theory of spontaneous parametric down-conversion has been presented using Maxwell's equations to describe electromagnetic wave propagation in quadratic non-linear uniaxial crystals. It has been shown that through SPDC, quantum entanglement can be achieved via a process that is simple to implement in a lab and can be understood using classical theory. The polarization entanglement of photons through SPDC has been shown to violate the Bell and Hardy inequalities, and throw localrealism into doubt. The simple SPDC methods for entanglement gives way to the possibility that light to matter entanglement transfer is the preferable method in creating stable and easily containable multiparticle entangled states, and thus simplifying the production of quantum computers. In general, it is difficult to localize and store photons, so usually one prefers choosing matter as quantum memory elements. The interface between quantum carrier and memory is a key component in the realization of scalable quantum networks. A scheme has been proposed to transfer entanglement from photons to nano-scale resonators constructed from movable mirror cavities, which oscillate at frequencies dependent upon the radiation pressure within the cavity when at cryogenic temperatures ${ }^{17}$. Each of the down-converted photons is absorbed into one of the mirror cavities (see Fig 11). This setup takes advantage of the superposition of frequency states of the signal and idler photons, so it is unknown which nanoresonator has absorbed $\omega_{1}$, and which has absorbed $\omega_{2}$. This causes the nanoresonators to be in a superposition of oscillation modes and be correlated with each other, and therefore be entangled.

The quantum computing industry could benefit from producing and transferring entanglement from light to matter using the relatively simple process of spontaneous parametric down-conversion. Further research is required to determine how to best utilize plasmons for quantum computing. Photons are the fastest carriers of quantum information for transmission, and the transmission of that information to matter could circumvent difficulties in localizing and storing photons for manipulation in quantum computers.

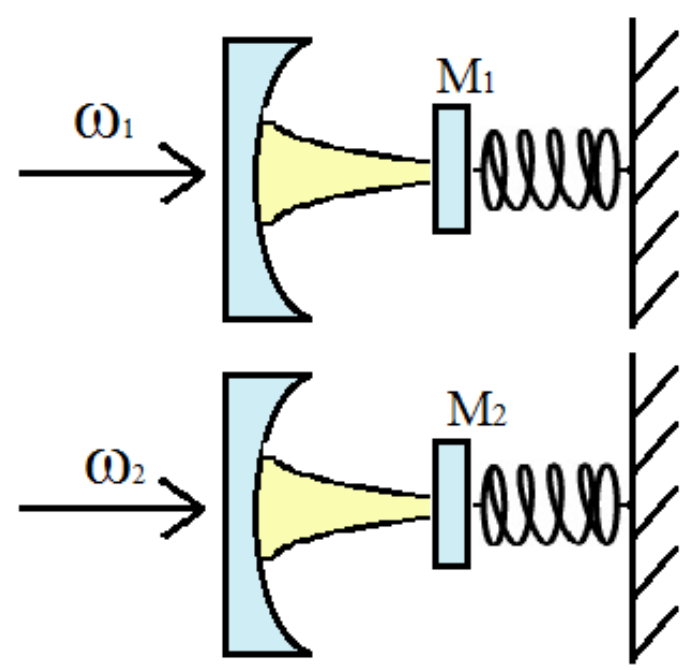

Fig 11: Two down-converted photons are each sent to and absorbed by a nanoresonating mirror cavity, which oscillate upon absorption. The mode of oscillation depends upon the energy (frequency) of the absorbed light. The two photons have frequencies that are related by $\omega_{3}=\omega_{1}+\omega_{2}$, but it is unknown which photon is of frequency $\omega_{1}$, and which is of $\omega_{2}$. Therefore, the mirror cavities $\mathrm{M}_{1}$ and $\mathrm{M}_{2}$ are entangled in their modes of oscillation. 


\section{Acknowledgements}

I give thanks to my advisor, Dr. La Rosa, for his guidance and supervision on this piece for the past year. His dedicated support, time, and resources have been a major factor in the completion of this thesis. I would also like to thank Mark Beck for providing insight into the nature of entanglement.

\section{Appendix - Vector Orientation Relative to the Principle Axis}

In the most general case, $\mathbf{E}$ and $\mathbf{D}$ in a non-linear material can be related by

$$
D_{j}=\sum_{i} \varepsilon_{\mathrm{ji}} \mathrm{E}_{\mathrm{i}} \quad, \text { where } \mathrm{j}, \mathrm{i}=1,2,3 \text {. }
$$

However, in the principle axis reference frame, the relationship can be written using the principal dielectric permittivities,

$$
\boldsymbol{D}=\left(\varepsilon_{\mathrm{x}} \mathrm{E}_{\mathrm{x}}, \varepsilon_{\mathrm{y}} \mathrm{E}_{\mathrm{y}}, \varepsilon_{\mathrm{z}} \mathrm{E}_{\mathrm{z}}\right)
$$

In this frame, $\mathbf{E}$ and $\mathbf{D}$ are always parallel when along the $\mathrm{x}, \mathrm{y}$, and $\mathrm{z}$ directions. In a uniaxial medium such as a KDP crystal $\left(\mathrm{KH}_{2} \mathrm{PO}_{4}\right)$, where $\varepsilon_{\mathrm{x}}=\varepsilon_{\mathrm{y}} \neq \varepsilon_{\mathrm{z}}$, the z-axis has a different index of refraction from the other two axes. Therefore, the behavior of light within a uniaxial medium will depend upon whether or not its polarization has a z-component. Because of this, the z-axis is often referred to as the extraordinary axis or optical axis. The indices are also given special names:

$$
\begin{array}{ll}
n_{o}=\sqrt{\frac{\varepsilon_{\mathrm{x}}}{\varepsilon_{0}}}=\sqrt{\frac{\varepsilon_{\mathrm{y}}}{\varepsilon_{0}}} & \text { Ordinary refractive index } \\
n_{e}=\sqrt{\frac{\varepsilon_{\mathrm{z}}}{\varepsilon_{0}}} & \text { Extraordinary refractive index }
\end{array}
$$

Let $\mathbf{E}$ and $\mathbf{D}$ be plane waves described by

$$
\begin{aligned}
& \boldsymbol{E}(\boldsymbol{r}, t)=\boldsymbol{E}_{0} e^{i(\boldsymbol{k} \cdot \boldsymbol{r}-\omega \mathrm{t})} \\
& \boldsymbol{D}(\boldsymbol{r}, t)=\boldsymbol{D}_{0} e^{i(\boldsymbol{k} \cdot \boldsymbol{r}-\omega \mathrm{t})}
\end{aligned}
$$

where $\mathbf{E}_{\mathrm{o}}$ and $\mathbf{D}_{\mathrm{o}}$ are constants, $\mathbf{k}$ is the wave vector, and $\omega$ is the frequency. Replacing (A-1) and (A-2) into equation (4) results in

$$
\boldsymbol{k} \times(\boldsymbol{k} \times \boldsymbol{E})=-\mu \omega^{2} \boldsymbol{D},
$$

which shows that $\mathbf{D}$ and $\mathbf{k}$ are perpendicular, and that $\mathbf{E}$ lies in the same plane as $\mathbf{D}$ and $\mathbf{k}$. If $\mathbf{E}$ and $\mathbf{D}$ lie in the xz-plane, then

$$
\begin{aligned}
& D_{x}=\varepsilon_{\mathrm{x}} \mathrm{E}_{\mathrm{x}}=\varepsilon_{0} n_{o}{ }^{2} E_{x} \\
& D_{z}=\varepsilon_{\mathrm{z}} \mathrm{E}_{\mathrm{z}}=\varepsilon_{0} n_{e}{ }^{2} E_{z} .
\end{aligned}
$$




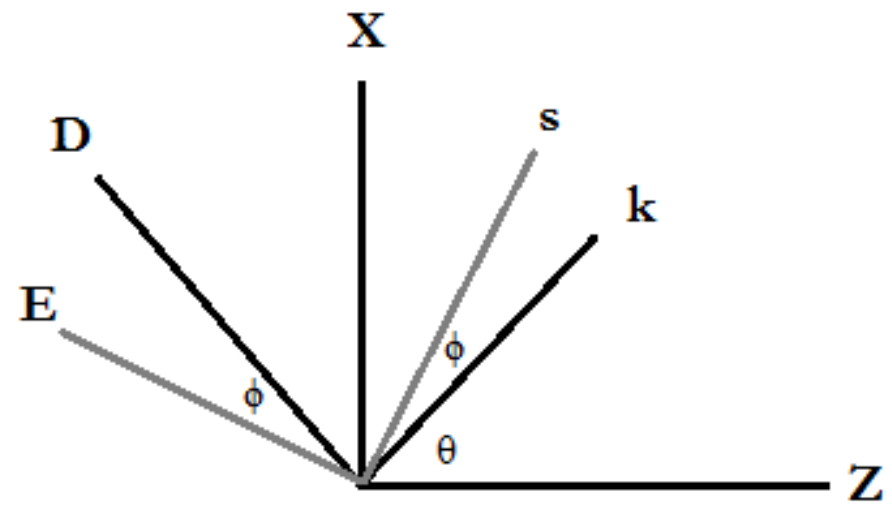

Figure A-1: Several vectors of an extraordinary wave in the principle axis reference frame. Angle $\square$ is between $\mathbf{E}$ and $\mathbf{D}$, and $\theta$ is between $\mathbf{k}$ and $\mathbf{z}$.

If $\mathbf{E}$ is an angle $\square$ from $\mathbf{D}$, and $\mathbf{k}$ is an angle $\theta$ from the optical axis as in Fig A-1, then

$$
\begin{aligned}
& \frac{D_{z}}{D_{x}}=-\tan \theta \\
& \frac{E_{z}}{E_{x}}=-\tan (\theta+\phi) .
\end{aligned}
$$

Replacing (A-3) and (A-4) into (A-5),

$$
\frac{E_{z}}{E_{x}}=-\frac{n_{o}^{2}}{n_{e}^{2}} \tan \theta
$$

Replacing (A-6) into (A-7), after some algebra, results in

$$
\phi=\tan ^{-1}\left(\frac{n_{o}^{2}}{n_{e}^{2}} \tan \theta\right)-\theta
$$

The values of the ordinary and extraordinary refractive indices can be calculated from the Sellmeier equations for KDP crystal (Kwiat, pg. 17) ${ }^{18}$,

$$
\begin{aligned}
& n_{o}{ }^{2}=2.259276+\frac{0.01008956}{\lambda^{2}-0.012942625}+\frac{\lambda^{2} 13.00522}{\lambda^{2}-400} \\
& n_{e}{ }^{2}=2.132668+\frac{0.008637494}{\lambda^{2}-0.012281043}+\frac{\lambda^{2} 3.2279924}{\lambda^{2}-400}
\end{aligned}
$$

where $\lambda$ is the wavelength of light in micrometers. The ratio of the squares of the refractive indices from (A-9) and (A-10) ranges from 1.05 to 1.06 when $\lambda$ is confined to the visible spectrum. In that case, according to (A-8), the value of $\phi$ is very small in KDP, with a maximum of about 1.67 degrees or 0.03 radians. This result is used in section 1.5 to approximate that the component of $\mathbf{k}$ in the direction of $\mathbf{E}$ is negligible when compared to the component of $\mathbf{k}$ in the direction of $\mathbf{s}$.

\section{References}

1. A. Zeilinger, (2010). "Dance of the Photons: From Einstein to Quantum Teleportaion”. New York: Straus and Giroux. Print. 
2. A. Zavatta, S. Viciani, and M. Bellini, (2004). "Tomographic reconstruction of the singlephoton Fock state by high-frequency homodyne detection”. Phys. Rev. A 70, 053821.

3. M. Beck, (2012). “Quantum Mechanics: Theory and Experiment”. Oxford, New York: Oxford University Press.

4. A. Yariv and P. Yeh, (2007). "Photonics: Optical Electronics in Modern Communications $6^{\text {th }}$ edition". Oxford, New York: Oxford University Press.

5. J. B. Pors, (2011). "Entangling Light in High Dimensions". Ph.D. thesis, Leiden University.

6. P.G. Kwiat, K. Mattle, H. Weinfurter, and A. Zeilinger, (1995). "New high-intensity source of polarization-entangled photons". Phys. Rev. A 75, 4337.

7. Y. Ono, T. Hikita, and T. Ikeda, (1987). "Phase Transitions in Mixed Crystal System $\mathrm{K}_{1-}$ ${ }_{x}\left(\mathrm{NH}_{4}\right)_{\mathrm{x}} \mathrm{H}_{2} \mathrm{PO}_{4} "$. Journal of the Physics Society Japan 56 (2), 577. doi:10.1143/JPSJ.56.577.

8. F. Zernike and J.E. Midwinter, (1973). “Applied Nonlinear Optics”. John Wiley \& Sons Inc.

9. R.W. Boyd, (1992). "Nonlinear Optics". San Diego, CA: Academic Press Inc.

10. R. Ramirez-Alarcon, H. Cruz-Ramirez and A. B. U'Ren, (2013). "Effects of crystal length on the angular spectrum of spontaneous parametric downconversion photon pairs". Laser Phys. DOI 23 :10.1088/1054-660X/23/5/055204.

11. B. John, (1964). "On the Einstein Podolsky Rosen Paradox". Physics 1 (3): 195-200.

12. L. Hardy, (1993). "Nonlocality for two particles without inequalities for almost all entangled states”. Phys. Rev. Lett. 71, 1665.

13. N. D. Mermin, (1994). “Quantum mysteries refined”. Am. J. Phys. 62, 880.

14. J.A.Carlson, M.D. Olmstead, and M. Beck, (2006). "Quantum mysteries tested: An experiment implementing Hardys test of local realism”, American Association of Physics Teachers DOI: 10.1119/1.2167764,

15. E. Altewischer, M.P. van Exter, and J.P. Woerdman, (2002). "Plasmon-assisted transmission of entangled photons" Nature 418, 304.

16. D. Stick, W. K. Hensinger, S. Olmschenk, M. J. Madsen, K. Schwab and C. Monroe, (2006). "Ion trap in a semiconductor chip". Nature Physics 2, 36-39.

17. J. Zhang, K. Peng, S. Braunstein, (2003). "Quantum-state transfer from light to macroscopic oscillators”. Phys. Rev. A 68, 013808.

18. P. G. Kwiat, (1993). "Nonclassical effects from spontaneous parametric down-conversion: Advetures in quantum wonderland". University of California, Berkely. 